\title{
Averaging for Switched DAEs: Convergence, Partial Averaging and Stability
}

\author{
Elisa Mostacciuolo ${ }^{\text {a }}$, Stephan Trenn ${ }^{\mathrm{b}}$, Francesco Vasca ${ }^{\mathrm{a}}$ \\ ${ }^{a}$ Department of Engineering, University of Sannio, 82100 Benevento, Italy \\ ${ }^{\mathrm{b}}$ Department of Mathematics, University of Kaiserslautern, 67663 Kaiserslautern, Germany
}

\begin{abstract}
Averaging is a useful technique to simplify the analysis of switched systems. In this paper we present averaging results for the class of systems described by switched differential algebraic equations (DAEs). Conditions on the consistency projectors are given which guarantee convergence towards a non-switched averaged system. A consequence of this result is the possibility to stabilize switched DAEs via fast switching. We also study partial averaging in case the consistency projectors do not satisfy the conditions for convergence; the averaged system is then still a switched system, but is simpler than the original. The practical interest of the theoretical averaging results is demonstrated through the analysis of the dynamics of a switched electrical circuit.
\end{abstract}

\section{Introduction}

Many dynamical systems present continuous and discrete behavior; they are called hybrid systems [3]. A switched system is a hybrid system consisting of a family of dynamical subsystems and a policy that at each time instant selects the active subsystem among a set of possible modes [8]. The selection policy is usually described by means of a switching function, which here is assumed to be a function of time (in contrast to state dependent switching).

In this paper we study switched systems whose modes are given by linear differential algebraic equations (DAEs). Linear DAEs are a natural way of modeling electrical circuit, simple mechanical systems or, in general, (linear) systems with additional (linear) algebraic constraints [7]. If this kind of systems change their model during the time one obtains a switched system; for example one can add (ideal) switches to an electrical circuit or allow for sudden structural changes in mechanical systems. The potentially complex interaction between the modes dynamics and the switching signal complicates the analysis of switched models. A possible approach to circumvent some of these difficulties, when switchings occur at high frequencies, is to average the hybrid dynamics over a time interval and to base the

\section{$\star$}

Email addresses: elisa.mostacciuolo@unisannio.it (Elisa Mostacciuolo), trenn@mathematik.uni-kl.de (Stephan Trenn), vasca@unisannio.it (Francesco Vasca). analysis and control design on the simpler averaged system.

Averaging theory for switched systems has a big interest in the control literature considering different approaches and points of view related to the switched system characteristics: non-periodic switching functions $[1,14]$, pulse modulations [17,12], dithering [4], effects of exogenous inputs [4], hybrid systems framework [21,22]. On the practical point of view, the averaging approach is a widely used technique in the power electronics community since 1970s [16,13] and has been also applied to other switched systems of practical interest, see [11] and the references therein.

This paper has three major contributions: 1) We establish an averaging result for linear switched DAEs, 2) we present a partial averaging result in case a smooth averaged model does not exist and 3) we show how the averaging result can be utilized to achieve stabilization via fast switching. The averaging result is based on the conference papers $[6,5]$, but we were able to considerable relax the assumptions on the consistency projectors. Commutativity is not necessary anymore; we consider for each consistency projector $\Pi_{i}$ related to the generic $i$-th mode, the Projector Assumption, (PA)

$$
\begin{aligned}
& \operatorname{im} \Pi_{\cap} \subseteq \operatorname{im} \Pi_{i}, \\
& \operatorname{ker} \Pi_{\cap} \supseteq \operatorname{ker} \Pi_{i},
\end{aligned}
$$

where $\Pi_{\cap}$ is the product of all the consistency projectors. The partial averaging result builds upon our confer- 
ence paper [10] which considers only two modes; here we present the result for arbitrarily many modes. The stabilization procedure for switched DAE is via fast switching and the averaging technique is new, but there is a strong connection to the results in [9]; in particular, $[9$, Rem. 21] already discusses this connection and concludes that the averaging technique may be more powerful because commutativity of the flows is not needed.

The paper is organized as following: in Section 2 we recall some mathematical notions, present some concepts regarding switched ordinary differential equations (ODEs) with jumps and some results from the theory of switched DAEs. In Section 3 we present the averaging result for switched DAEs; the stability analysis is carried out in Section 4 resulting in a method for stabilization via fast switching. In Section 5 the partial averaging result is presented. The conclusions of the work are summarized in Section 6.

\section{Switched ODEs and switched DAEs}

In the following subsections some preliminary definitions are recalled. Furthermore, in order to present the averaging technique, some results regarding the switched ODEs and some concept of the theory of switched DAEs are illustrated. In the sequel the following notation is adopted: $\mathbb{R}^{n}$ is the set of $n$-th dimensional real vectors, $\mathbb{R}_{+}$is the set of nonnegative real numbers, $\mathbb{N}$ is the set of nonnegatives integers, the product of any q matrices $\left\{M_{i}\right\}_{i=1}^{\mathrm{q}}$ is defined as (note the order)

$$
\prod_{i=1}^{\mathrm{q}} M_{i}=M_{\mathrm{q}} M_{\mathrm{q}-1}, \ldots, M_{2} M_{1}
$$

$\|\cdot\|$ is the Euclidean norm and $\|\cdot\|_{\infty}$ is the infinity norm.

Definition 1 (Lipschitz function) A function $f(p)$ : $\mathbb{R}_{+} \rightarrow \mathbb{R}^{n}$ is Lipschitz if there exists a positive constant $L$ such that $\forall p_{1}, p_{2}$ the inequality

$$
\left\|f\left(p_{1}\right)-f\left(p_{2}\right)\right\| \leq L\left\|p_{1}-p_{2}\right\|
$$

holds.

\subsection{Big-O notation}

Definition 2 (Big-O notation) Given any functions $f(p): \mathbb{R}_{+} \rightarrow \mathbb{R}^{n}$ and $g(p): \mathbb{R}_{+} \rightarrow \mathbb{R}_{+}$, we say that $f(p)$ is an $\mathrm{O}(g(p))$ function as $p \rightarrow 0(f(p)=\mathrm{O}(g(p))$ for short), if there exist positive constants $\alpha$ and $\bar{p}$ such that

$$
\|f(p)\| \leq \alpha g(p), \quad \forall p \in[0, \bar{p}]
$$

When $g(p)$ is the identity function we indicate $f(p)=$ $\mathrm{O}(p)$. Clearly any linear combination of functions which are $\mathrm{O}(p)$ is an $\mathrm{O}(p)$ itself. Moreover if $f(p)$ is Lipschitz then it is also $\mathrm{O}(p)$ but the converse does not necessarily hold because Definition 2 does not require $f(p)$ to be continuous. If $f(p)=\mathrm{O}(p)$ than it $f(0) \rightarrow 0$ as $p \rightarrow 0$. Given a compact set $\mathfrak{I} \subset(0, \infty)$, we say that

$$
f(t)-g(t)=\mathrm{O}(p), \quad \forall t \in \mathfrak{I}
$$

if the difference of two functions is $\mathrm{O}(p)$ uniformly in $t$, i.e. the constant $\alpha$ is independent of $t$.

By considering the Taylor approximation we can write, for any matrix $M \in \mathbb{R}^{n \times n}$ and any $s \in[0, p]$

$$
e^{M s}=I+M s+\mathrm{O}\left(p^{2}\right)=I+\mathrm{O}(p)
$$

where $I$ is the identity matrix.

\subsection{Definition and properties of projectors}

Definition 3 (Projector) Let $x \in \mathbb{R}^{n}=V \bigoplus W$. The linear operator $\rho_{V, W}: \mathbb{R}^{n} \rightarrow \mathbb{R}^{n}$ defined by

$$
\rho_{V, W}(v+w)=v
$$

where $v \in V$ and $w \in W$ is called projection of $x=v+w$ onto $V$ along $W$. The corresponding matrix $\Pi \in \mathbb{R}^{n \times n}$, defined by $v=\Pi x$ is called projector onto $V$ along $W$.

Note that a matrix $\Pi \in \mathbb{R}^{n \times n}$ is a projector if and only if it is idempotent, i.e., $\Pi^{2}=\Pi[15$, Thm. 2.21].

Lemma 4 Let $\Pi \in \mathbb{R}^{n \times n}$ be a projector and $M \in \mathbb{R}^{n \times n}$ then

$$
\begin{array}{rlll}
\operatorname{im} M \subseteq \operatorname{im} \Pi & \Leftrightarrow & \Pi M=M, \\
\operatorname{ker} M \supseteq \operatorname{ker} \Pi & \Leftrightarrow & M \Pi=M .
\end{array}
$$

PROOF. Necessity in both case is trivial. Since $\Pi$ is the identity on im $\Pi$ sufficiency for the first case is also clear. Considering the transpose and orthogonal complements, sufficiency of the second case follows with analogous arguments.

Corollary 5 If a family of projectors $\left\{\Pi_{i}\right\}_{i=1}^{q}$ with

$$
\Pi_{\cap}:=\prod_{i=1}^{\mathrm{q}} \Pi_{i}
$$

satisfies the Projector Assumption (PA) then $\Pi_{\cap}^{2}=\Pi_{\cap}$, i.e. $\Pi_{\cap}$ itself is a projector.

Lemma 6 ([5, Lem. 2 \& Lem. 3]) Let $\ell(p) \in \mathbb{R}_{+} \rightarrow$ $\mathbb{N}$ be such that $p \ell(p)=\mathrm{O}(1)$ and let $\Pi: \mathbb{R}^{n \times n}$ be a projector. Then

$$
(\Pi+\mathrm{O}(p))^{\ell(p)}=\mathrm{O}(1)
$$




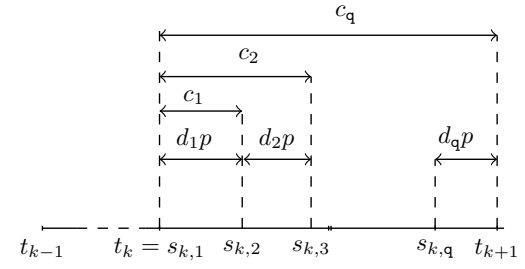

Fig. 1. Graphical representation of the time interval $\left[t_{k-1}, t_{k+1}\right]$, with $t_{k}=k p, t_{k+1}=k p+p$, $s_{k, i}:=k p+\sum_{j=1}^{i-1} d_{j} p$ and $c_{i}:=\sum_{j=1}^{i} d_{j} p i=2, \ldots, \mathrm{q}$.

Furthermore, for any matrices $M, \tilde{M} \in \mathbb{R}^{n \times n}$ with

$$
\Pi M \Pi=M=\Pi \tilde{M} \Pi
$$

it holds that

$$
\begin{aligned}
\Pi\left(\left(\Pi+\tilde{M} p+O\left(p^{2}\right)\right)^{\ell(p)}-(\Pi+M p\right. \\
\left.\left.\quad+O\left(p^{2}\right)\right)^{\ell(p)}\right) \Pi=\ell(p) O\left(p^{2}\right) .
\end{aligned}
$$

An interesting interpretation for $\ell(p)$ in Lemma 6 is the number of consecutive periods of length $p$ inside a fixed time interval $[0, \Delta]$. Indeed for this case $\ell(p)$ tends to infinity when $p$ goes to zero but $p \ell(p)=\mathrm{O}(1)$.

\subsection{Switched ODEs}

Let $\sigma: \mathbb{R}_{+} \rightarrow \Sigma$ be a piecewise constant rightcontinuous function, that selects at each time instant the index of the active mode from the finite index set $\Sigma:=\{1,2, \ldots, \mathrm{q}\}$. In the sequel $\sigma$ is called the switching signal. Here we assume that $\sigma$ is periodic with switching period $p>0$. The switching time instants are defined as follows

$$
t_{k}:=k p, k \in \mathbb{N}, \quad s_{k, i}:=t_{k}+\sum_{j=1}^{i-1} d_{j} p, i \in \Sigma,
$$

where $d_{i} \in(0,1)$ is the duty cycle of the $i$-th mode, see Figure 1 ; in particular $\sum_{i=1}^{\mathrm{q}} d_{i}=1$. Note that $s_{k, 1}=t_{k}$.

Then the switching signal can be written as

$$
\sigma(t)=\left\{\begin{array}{cl}
1, & t \in\left[t_{k}, s_{k, 2}\right) \\
2, & t \in\left[s_{k, 2}, s_{k, 3}\right), \\
\vdots & \\
\mathrm{q}, & t \in\left[s_{k, \mathrm{q}}, t_{k+1}\right) .
\end{array}\right.
$$

Let $c_{i}$ be the time interval between the beginning of any period and the end of the $i$-th mode, i.e.

$$
c_{i}:=\sum_{j=1}^{i} d_{j} p, \quad i \in \Sigma
$$

Note that in the sequel we use $c_{i}$ with $i=0, \ldots, \mathrm{q}$ where we assume $c_{0}=0$, obviously it is $c_{q}=p$.

A switched ODE with switching signal (5)-(6) and continuous input $u: \mathbb{R}_{+} \rightarrow \mathbb{R}^{m}$ is given by

$$
\dot{w}(t)=A_{\sigma(t)} w(t)+B_{\sigma(t)} u(t), \quad \forall t \in \mathbb{R}_{+}
$$

with initial condition $w\left(0^{-}\right)=w_{0}$.

The averaged model for switched ODEs is given by

$$
\dot{w}_{\mathrm{av}}(t)=\sum_{i=1}^{\mathrm{q}} d_{i}\left(A_{i} w_{\mathrm{av}}(t)+B_{i} u(t)\right)
$$

where $A_{i} \in \mathbb{R}^{n \times n}$ and $B_{i} \in \mathbb{R}^{n \times m}$, see [13].

Dealing with the approximation properties between the averaged system and the switched system, it is possible to show that the approximation is of order of the period $p$ assuming that the two systems have the same initial condition $w_{0}$ and that the exogenous input $u$ is bounded, differentiable and with bounded derivative, [12]. No further assumptions on the matrices $A_{i}$ and $B_{i}$ are needed for this approximation result.

\subsection{Switched ODEs with jumps}

The averaging analysis for switched DAEs which we present in the next sections is based on some properties of the solution of switched ODEs where the state contains jumps.

A switched ODE with switching signal (5)-(6), continuous input $u: \mathbb{R}_{+} \rightarrow \mathbb{R}^{m}$, jump input $v: \mathbb{R}_{+} \rightarrow \mathbb{R}^{n}$ and discontinuous state variable $w \in \mathbb{R}^{n}$ is given by

$$
\begin{aligned}
\dot{w}(t) & =A_{\sigma(t)} w(t)+B_{\sigma(t)} u(t), \quad \forall t \neq s_{k, i} \wedge t \in \mathbb{R}_{+} \\
w\left(s_{k, i}^{+}\right) & =\Pi_{\sigma\left(s_{k, i}\right)} w\left(s_{k, i}^{-}\right)+Q_{\sigma\left(s_{k, i}\right)} v\left(s_{k, i}\right),
\end{aligned}
$$

with initial condition $w\left(0^{-}\right)=w_{0} \in \mathbb{R}^{n}, k \in \mathbb{N}$, and $i \in \Sigma$. The first equation in (9) describes the dynamics in the different modes, while the second equation represents the jump rule at the switching time instants.

The switched ODE (9) can be rewritten in terms of the modes

$$
\begin{aligned}
\dot{w}(t) & =A_{i} w(t)+B_{i} u(t), \quad t \in\left(s_{k, i}, s_{k, i+1}\right) \\
w\left(s_{k, i}^{+}\right) & =\Pi_{i} w\left(s_{k, i}^{-}\right)+Q_{i} v_{s_{k, i}}, \\
w\left(0^{-}\right) & =w_{0}
\end{aligned}
$$


with $v_{s_{k, i}}=v\left(s_{k, i}\right), k \in \mathbb{N}, i \in \Sigma$, the switching times given by (5) and matrices $A_{i} \in \mathbb{R}^{n \times n}, B_{i}, Q_{i} \in \mathbb{R}^{n \times m}$ and the projectors $\Pi_{i} \in \mathbb{R}^{n \times n}$.

In the following Lemma we express the solution of (9) evaluated at the multiplies of the switching period in a compact form depending on the initial conditions and on the input.

Lemma 7 Consider the switched ODE (9) and switching times given by (5). There exist matrices $H(p), N(p)$ and an operator $\mathcal{I}(p)$ such that every solution of $(9)$ satisfies

$w\left(t_{k}^{-}\right)=H(p) w\left(t_{k-1}^{-}\right)+N(p) v_{k-1}+\mathcal{I}(p)\left\{u_{k-1}\right\} \quad \forall k \in \mathbb{N}$

where $u_{k-1}$ indicates the input function on the time interval $\left(t_{k-1}, t_{k}\right)$ translated into the time interval $(0, p)$ :

$$
u_{k-1}:[0, p] \rightarrow \mathbb{R}^{m}, \xi \mapsto u\left(\xi+t_{k-1}\right),
$$

and

$$
v_{k-1}:=\left[\begin{array}{c}
v_{s_{k-1,1}} \\
v_{s_{k-1,2}} \\
\vdots \\
v_{s_{k-1, q}}
\end{array}\right]
$$

In particular,

$w\left(t_{k}^{-}\right)=H(p)^{k} w_{0}+\sum_{i=0}^{k-1} H(p)^{k-1-i}\left(N(p) v_{i}+\mathcal{I}(p)\left\{u_{i}\right\}\right)$

PROOF. The solution of the switched ODE on the interval $\left(s_{k-1, \mathrm{q}}, t_{k}\right)$ evaluated at $t_{k}^{-}$is given by

$$
w\left(t_{k}^{-}\right)=e^{A_{\mathrm{q}} d_{\mathrm{q}} p} w\left(s_{k-1, \mathrm{q}}^{+}\right)+\int_{s_{k-1, \mathrm{q}}}^{t_{k}} e^{A_{\mathrm{q}}\left(t_{k}-\xi\right)} B_{\mathrm{q}} u(\xi) \mathrm{d} \xi .
$$

Furthermore

$$
w\left(s_{k-1, \mathrm{q}}^{+}\right)=\Pi_{\mathrm{q}} w\left(s_{k-1, \mathrm{q}}^{-}\right)+Q_{\mathrm{q}} v_{s_{k-1, \mathrm{q}}}
$$

where $w\left(s_{k-1, q}^{-}\right)$is the solution on the interval $\left(s_{k-1, \mathrm{q}-1}, s_{k-1, \mathrm{q}}\right)$ evaluated at $s_{k-1, \mathrm{q}}^{-}$.

Substituting the solution $w\left(s_{k-1, \mathrm{q}}^{-}\right)$in (13) and then in (12), and by iterating for all q modes one obtains the linear discrete time system

$$
w\left(t_{k}^{-}\right)=H(p) w\left(t_{k-1}^{-}\right)+N(p) v_{k-1}+\mathcal{I}(p)\left\{u_{k-1}\right\}
$$

with solution (11), where

$$
\begin{aligned}
H(p)= & \prod_{i=1}^{\mathrm{q}} e^{A_{i} d_{i} p} \Pi_{i} \\
\mathcal{I}(p)\left\{u_{k-1}\right\}= & \sum_{i=1}^{\mathrm{q}} \prod_{j=i+1}^{\mathrm{q}} e^{A_{j} d_{j} p} \Pi_{j} \times \\
& \times \int_{c_{i-1}}^{c_{i}} e^{A_{i}\left(c_{i}-\xi\right)} B_{i} u_{k-1}(\xi) \mathrm{d} \xi \\
N(p)= & {\left[\prod_{i=1}^{\mathrm{q}}\left(e^{A_{i} d_{i} p} \Pi_{i}\right) Q_{1} \prod_{i=2}^{\mathrm{q}}\left(e^{A_{i} d_{i} p} \Pi_{i}\right) Q_{2}\right.} \\
& \left.e^{A_{\mathrm{q}} d_{\mathrm{q}} p} Q_{\mathrm{q}}\right]
\end{aligned}
$$

and $c_{i}$ are given by (7) with $i \in \Sigma$.

The solution of (9) with $w_{0}=0$, under some conditions, is an $\mathrm{O}(p)$ function as shown in the following Lemma.

Lemma 8 Consider the switched ODE (9) with initial condition $w\left(0^{-}\right)=0$ and switching times given by (5). Consider any given interval $[0, \Delta]$ where $\Delta \in \mathbb{R}_{+}$, and assume that the following conditions hold

(i) $u(t)=\mathrm{O}(p), \forall t \in[0, \Delta]$,

(ii) $v_{s_{k, i}}=O(p), \forall k \in \mathbb{N}, i \in \Sigma$,

(iii) $\Pi_{\cap}$ given by $(2)$, is a projector,

(iv) $\Pi_{i} Q_{i-1}=0, i \in \Sigma$ with $Q_{0}:=Q_{\mathrm{q}}$.

Then $w(t)=\mathrm{O}(p), \forall t \in[0, \Delta]$.

PROOF. The solution of (9) is given by (11) where $w_{0}=0$. Let $\ell(p)$ be the number of consecutive periods of length $p$ inside $[0, \Delta]$, i.e., $\Delta-p<p \ell(p) \leq \Delta$. Note that $p \ell(p)=\mathrm{O}(1)$. Taking into account that $\Pi_{1}^{2}=\Pi_{1}$, the expression (11) can be rewritten as

$$
\begin{aligned}
w\left(t_{k}^{-}\right)= & \sum_{i=0}^{k-2} H(p)^{k-1-i}\left(\Pi_{1} N(p) v_{i}+\Pi_{1} \mathcal{I}(p)\left\{u_{i}\right\}\right) \\
& +N(p) v_{k-1}+\mathcal{I}(p)\left\{u_{k-1}\right\}
\end{aligned}
$$

for $k=2, \ldots, \ell(p)$ and the same expression without the first sum for $k=1$. By using (1) in (15a) and (15c) we obtain

$$
\begin{aligned}
& H(p)=\Pi_{\cap}+\mathrm{O}(p), \\
& N(p)=\left[\Pi_{\mathrm{q}} \Pi_{\mathrm{q}-1} \cdots \Pi_{2} Q_{1}+\mathrm{O}(p) \ldots Q_{\mathrm{q}}+\mathrm{O}(p)\right],
\end{aligned}
$$

and by invoking the assumption (iv),

$$
N(p)=\mathrm{O}(p) .
$$


Furthermore, invoking (iii) and (3),

$$
H(p)^{k-1}=\mathrm{O}(1), \quad k=1, \ldots, \ell(p) .
$$

Finally, taking into account the general bound $\left\|\int_{a}^{b} f\right\| \leq$ $(b-a)\|f\|_{\infty}$ and using (1) in (15b), it follows

$$
\mathcal{I}(p)\left\{u_{i}\right\}=\mathrm{O}(p)\left\|u_{i}\right\|_{\infty}=\mathrm{O}\left(p^{2}\right), \quad i=0, \ldots, \ell(p)-1
$$

where we also used (i).

Hence it follows, together with assumptions (i) and (ii),

$$
w\left(t_{k}^{-}\right)=(k-1) \mathrm{O}\left(p^{2}\right)+\mathrm{O}\left(p^{2}\right),
$$

for $k=1, \ldots, \ell(p)$. Since $\ell(p) \mathrm{O}\left(p^{2}\right)=\mathrm{O}(p)$ from the equation above we obtain

$$
w\left(t_{k}^{-}\right)=\mathrm{O}(p) \text { as well as } w\left(t_{k}^{+}\right)=\mathrm{O}(p),
$$

for $k=1, \ldots, \ell(p)$. It remains to be shown that $w(t)=$ $\mathrm{O}(p)$ for $t \in\left(t_{k}, t_{k+1}\right)$ with $k=1, \ldots, \ell(p)$. The solution of (9) for any $\tau \in\left[s_{k, i}, s_{k, i+1}\right)$ and for any $i \in \Sigma$ can be written as follows

$$
w(\tau)=e^{A_{i}\left(\tau-s_{k, i}\right)} w\left(s_{k, i}^{+}\right)+\int_{s_{k, i}}^{\tau} e^{A_{i}(\tau-\xi)} B_{i} u(\xi) \mathrm{d} \xi .
$$

Considering the Taylor expression (1) with $s=\tau-s_{k, i}$ and by applying (i) we have

$$
w(\tau)=(I+\mathrm{O}(p)) w\left(s_{k, i}^{+}\right)+\mathrm{O}\left(p^{2}\right)=w\left(s_{k, i}^{+}\right)+\mathrm{O}(p) .
$$

By concatenating (17) for increasing values of $i \in \Sigma$ and by using (16) together with

$$
\begin{aligned}
w\left(s_{k, i}^{+}\right) & =\Pi_{i} w\left(s_{k, i}^{-}\right)+Q_{i} v_{s_{k, i}} \\
& =\Pi_{i} w\left(s_{k, i}^{-}\right)+\mathrm{O}(p),
\end{aligned}
$$

$\forall i \in \Sigma$; we obtain that $w(\tau)=\mathrm{O}(p) \forall \tau \in\left[t_{k}, t_{k+1}\right)$ and $k=1, \ldots, \ell(p)$, which completes the proof.

Remark 9 Lemma 8 is similar to classical input-tostate-stability (ISS) results, in the sense that a small input (of order $O(p)$ ) results in a small state $($ also $\mathrm{O}(p)$ ) on any fixed time interval. Recently, an ISS result utilizing averaging for general hybrid systems has been investigated [22].

\subsection{Switched DAEs}

A non-homogeneous switched linear DAE is given by

$$
E_{\sigma(t)} \dot{x}(t)=A_{\sigma(t)} x(t)+B_{\sigma(t)} u(t), \quad t \in \mathbb{R}_{+}
$$

where $x: \mathbb{R}_{+} \rightarrow R^{n}$ is the state, $u: \mathbb{R}_{+} \rightarrow \mathbb{R}^{m}$ is the Lipschitz continuous input, $x\left(0^{-}\right)=x_{0}$ is the initial condition and the switching signal $\sigma$ is defined as in (5)(6).

The dynamic of each mode $i$ of the system is given by the following linear DAE

$$
E_{i} \dot{x}(t)=A_{i} x(t)+B_{i} u(t)
$$

where $E_{i}, A_{i} \in \mathbb{R}^{n \times n}, B_{i} \in \mathbb{R}^{n \times m}$ are constant matrices for each $i \in \Sigma$. All solutions of each mode evolve within a consistency space that is a linear subspace of $\mathbb{R}^{n}$. The value $x\left(s_{k, i}^{-}\right)$just before a switching instant $s_{k, i}$ is not necessarily in the consistency space of the mode after the switch. Therefore it is necessary to allow solutions with jumps; this leads to problems in evaluating the derivative in (18). To resolve this problem we use the distributional solution framework [18]. Furthermore, the solutions of switched DAE can also contain Dirac impulses (in addition to possible jumps), but in this paper we only consider the impulse-free part of the solution (jumps are still possible). Recently, some preliminary results concerning the convergence of the Dirac impulses were obtained [19].

If the matrix pairs $\left(E_{i}, A_{i}\right)$ are regular, i.e. $m=n$ and the polynomial $\operatorname{det}\left(s E_{i}-A_{i}\right)$ is not the zero polynomial, then the following result is well known:

Proposition 10 (Quasi-Weierstrass form) $A$ matrix pair $(E, A) \in \mathbb{R}^{n \times n} \times \mathbb{R}^{n \times n}$ is regular if and only if there exist invertible transformation matrices $S, T \in \mathbb{R}^{n \times n}$ which put $(E, A)$ into quasi Weierstrass form

$$
(S E T, S A T)=\left(\left[\begin{array}{ll}
I & 0 \\
0 & N
\end{array}\right],\left[\begin{array}{ll}
J & 0 \\
0 & I
\end{array}\right]\right)
$$

where $N \in \mathbb{R}^{n_{2} \times n_{2}}$, with $0 \leq n_{2} \leq n$ is a nilpotent matrix, $J \in \mathbb{R}^{n_{1} \times n_{1}}$ with $n_{1}=n-n_{2}$ is some matrix and $I$ is the identity matrix of the appropriate size.

Note that, the transformation matrices $S$ and $T$ can easily be obtained via the so called Wong sequences, see $[2]$.

Definition 11 (Consistency projector, flow matrix) Consider a regular matrix pair $(E, A)$ and its quasi Weierstrass form (20). The consistency projector $\Pi$ of $(E, A)$ is given by

$$
\Pi=T\left[\begin{array}{ll}
I & 0 \\
0 & 0
\end{array}\right] T^{-1}
$$


and the so called flow matrix $A^{\operatorname{diff}}$ of $(E, A)$ is given by

$$
A^{\mathrm{diff}}=T\left[\begin{array}{ll}
J & 0 \\
0 & 0
\end{array}\right] T^{-1}
$$

Note that the flow matrix and the projectors do not depend on the specific choice of $T$, furthermore it is easily seen that $\Pi^{2}=\Pi$ and

$$
A^{\text {diff }} \Pi=A^{\text {diff }}=\Pi A^{\text {diff }} .
$$

Definition 12 (Differential and impulse projectors) Consider a regular matrix pair $(E, A)$ and its quasi Weierstrass form (20). The differential projector $\Pi$ diff of $(E, A)$ is given by

$$
\Pi^{\mathrm{diff}}=T\left[\begin{array}{ll}
I & 0 \\
0 & 0
\end{array}\right] S
$$

and the impulsive projector $\Pi^{\mathrm{imp}}$ of $(E, A)$ is given by

$$
\Pi^{\mathrm{imp}}=T\left[\begin{array}{ll}
0 & 0 \\
0 & I
\end{array}\right] S
$$

Note that the differential and impulsive projectors do not depend on the specific choice of $T$ and of $S$, and they are in general not idempotent matrices.

The role of projectors and the flow matrix becomes clear with the following result.

Theorem 13 Consider the switched DAE (18) with regular matrix pairs $\left(E_{i}, A_{i}\right)$ and corresponding flow matrices $A_{i}^{\text {diff }}$ and projectors $\Pi_{i}, \Pi_{i}^{\mathrm{imp}}, \Pi_{i}^{\mathrm{diff}}$ for $i \in \Sigma$. Assume that

$$
\Pi_{i}^{\mathrm{imp}} B_{i}=0, \quad \forall i \in \Sigma .
$$

Then $x: \mathbb{R} \rightarrow \mathbb{R}^{n}$ is the impulse free part of any (distributional) solution of (18) if and only if $x$ is a solution of the switched ODE with jumps given by

$$
\begin{aligned}
\dot{x}(t) & =A_{i}^{\text {diff }} x(t)+B_{i}^{\text {diff }} u(t), \quad \forall t \in\left(s_{k, i}, s_{k, i+1}\right) \\
x\left(s_{k, i}^{+}\right) & =\Pi_{i} x\left(s_{k, i}^{-}\right), \\
x\left(0^{-}\right) & =x_{0},
\end{aligned}
$$

where $B_{i}^{\text {diff }}:=\Pi_{i}^{\text {diff }} B_{i}, i \in \Sigma, k \in \mathbb{N}$.

PROOF. The (impulse-free) solution of (18) is obtained by "concatenating" the solution of each mode (19), that can be written as follows

$$
\begin{aligned}
x(t)=e^{A_{i}^{\mathrm{diff}}\left(t-s_{k, i}\right)} x\left(s_{k, i}^{+}\right) & +\int_{s_{k, i}}^{t} e^{A_{i}^{\mathrm{diff}}(t-s)} \Pi_{i}^{\mathrm{diff}} B_{i} u(s) \mathrm{d} s \\
& -\sum_{i=0}^{n-1}\left(E_{i}^{\mathrm{imp}}\right)^{i} \Pi_{i}^{\mathrm{imp}} B_{i} u(t)^{(i)}
\end{aligned}
$$

with $E_{i}^{\mathrm{imp}}:=\Pi_{i}^{\mathrm{imp}} E_{i}$ and $t \in\left(s_{k, i}, s_{k, i+1}\right)$. By using (22), the initial condition of the $i$-th mode is given by the second equation in $(23)$, where $x\left(s_{k, i}^{-}\right)$is the solution of mode $i-1$ evaluated at the time instant time $s_{k, i}^{-}$. Then the proof directly follows by considering (24) combined with (22).

Remark 14 Consider the switched DAE (18) with regular matrix pairs $\left(E_{i}, A_{i}\right)$ and corresponding flow matrices $A_{i}^{\text {diff }}$ and consistency projectors $\Pi_{i}$ for $i \in \Sigma$. Assume that $u(t)=0 \forall t$, i.e. (18) is an homogeneous switched $D A E$. Then $x$ is the impulse free part of any (distributional) solution of (18) if and only if $x$ is a solution of the switched ODE with jumps given by Theorem 13 where $u(t)=0 \forall t,[20]$.

Remark 15 By using the same arguments of the previous section we can write the solution of the switched $O D E(23)$ at $_{k}$ in a form similar to (11) with $v_{i}=0 \forall i \in$ $\mathbb{N}$; in particular

$$
x\left(t_{k}^{-}\right)=H_{\mathrm{diff}}(p)^{k} w_{0}+\sum_{i=0}^{k-1} H_{\mathrm{diff}}(p)^{k-1-i} \mathcal{I}_{\mathrm{diff}}(p)\left\{u_{i}\right\} .
$$

where

$$
\begin{aligned}
H_{\mathrm{diff}}(p)= & \prod_{i=1}^{q} e^{A_{i}^{\mathrm{diff}} d_{i} p} \Pi_{i} \\
\mathcal{I}_{\mathrm{diff}}(p)\left\{u_{k-1}\right\}= & \sum_{i=1}^{\mathrm{q}} \prod_{j=i+1}^{\mathrm{q}} e^{A_{j}^{\mathrm{diff}} d_{j} p} \Pi_{j} \times \\
& \times \int_{c_{i-1}}^{c_{i}} e^{A_{i}^{\text {diff }}\left(c_{i}-\xi\right)} B_{i}^{\mathrm{diff}} u_{k-1}(\xi) \mathrm{d} \xi
\end{aligned}
$$

with $c_{i}$ given by $(7)$.

\section{Averaging for switched DAEs}

Averaging theory is based on the observation that a rapidly time-varying system can be viewed as a small perturbation of a simplified, time-invariant, averaged system.

Given a switched DAE (18) with periodic switching signal $\sigma$ with period $p>0$ defined as in (5)-(6), if the 
switching signal is faster than the variations of the continuous state space variable $x$, we could try to investigate the possible existence of an averaged model. The averaged system approximates the behavior of the system; to the limit $p \rightarrow 0$ the solution of the averaged model converges to that of the switched system, i.e., the error between the solutions of the averaged and switched models is $\mathrm{O}(p)$.

We want to find conditions such that an averaged model of (18) is given by

$$
\begin{aligned}
\dot{x}_{\mathrm{av}}(t) & =A_{\mathrm{av}} x_{\mathrm{av}}(t)+B_{\mathrm{av}} u(t), \quad t \in \mathbb{R}_{+} \\
x_{\mathrm{av}}(0) & =\Pi_{\cap} x_{0}
\end{aligned}
$$

where

$$
\left.\begin{array}{rl}
A_{\mathrm{av}} & :=\Pi_{\cap} A_{\mathrm{av}}^{\mathrm{diff}} \Pi_{\cap} \\
B_{\mathrm{av}} & :=\Pi_{\cap} B_{\mathrm{av}}^{\mathrm{diff}} \\
A_{\mathrm{av}}^{\mathrm{diff}} & :=\sum_{i=1}^{\mathrm{q}} d_{i} A_{i}^{\mathrm{diff}} \\
B_{\mathrm{av}}^{\mathrm{diff}} & :=\sum_{i=1}^{\mathrm{q}} d_{i} B_{i}^{\mathrm{diff}}
\end{array}\right\}
$$

where $d_{i}, i \in \Sigma$, is the duty cycle of the $i$-th mode defined by (5) and $\Pi_{\cap}$ is given by (2). The expressions (28) have an elegant parallelism with the matrices in (8).

The solution of the averaged model at the multiples of the period can be easily written as in (11)

$$
x_{\mathrm{av}}\left(t_{k}^{-}\right)=H_{\mathrm{av}}(p)^{k} \Pi_{\cap} x_{0}+\sum_{i=0}^{k-1} H_{\mathrm{av}}(p)^{k-1-i} \mathcal{I}_{\mathrm{av}}(p)\left\{u_{i}\right\}
$$

with

$$
\begin{aligned}
H_{\mathrm{av}}(p) & =e^{A_{\mathrm{av}} p} \\
\mathcal{I}_{\mathrm{av}}(p)\left\{u_{k-1}\right\} & =\int_{0}^{p} e^{A_{\mathrm{av}}(p-\xi)} B_{\mathrm{av}} u_{k-1}(\xi) \mathrm{d} \xi
\end{aligned}
$$

Remark 16 The solution of the averaged model (27)(28) belongs to $\mathrm{im} \Pi_{\cap}$. Then if (PA) hold $\forall i \in \Sigma$ and taking into account Lemma 4, we have

$$
x_{a v}(t)=\Pi_{i} x_{a v}(t), \forall t \in \mathbb{R}_{+}, \forall i \in \Sigma .
$$

In the following subsections we present conditions for which (27)-(28) represent an averaged model of (18) for the homogeneous and non-homogeneous cases, respectively.

\subsection{Homogeneous switched DAEs}

In the following example we consider a switched DAE (18) with $u(t)=0 \forall t$.
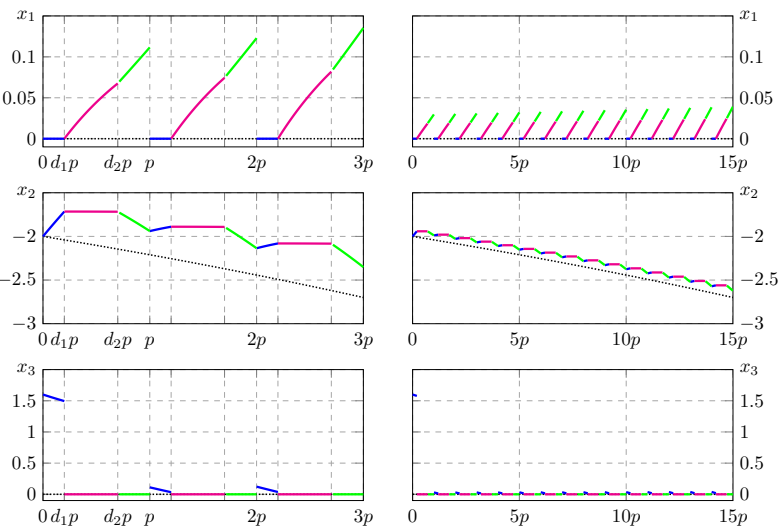

Fig. 2. Evolution of state variables (first component top, second component middle, third component bottom) of Example 17 for slow switching ( $p=0.1 s$, left) and fast switching $(p=0.02 s$, right $)$. The averaging dynamics are plotted with dotted black lines, while the trajectories of the switched DAE are colored according to the active mode (mode 1 blue, mode 2 magenta, mode 3 green). Note that $x_{3}$ is not $\mathrm{O}(p)$ on $[0, p)$.

Example 17 Consider the following matrices

$$
\begin{array}{ll}
E_{1}=\left[\begin{array}{lll}
0 & 1 & 0 \\
1 & 0 & 1 \\
0 & 0 & 0
\end{array}\right], & A_{1}=\left[\begin{array}{ccc}
8 & -1 & 8 \\
-1 & 2 & -1 \\
1 & 0 & 0
\end{array}\right], \\
E_{2}=\left[\begin{array}{lll}
1 & 0 & 0 \\
0 & 1 & 0 \\
0 & 0 & 0
\end{array}\right], & A_{2}=\left[\begin{array}{ccc}
-10 & -1 & -10 \\
-1 & 0 & -1 \\
0 & 0 & 1
\end{array}\right], \\
E_{3}=\left[\begin{array}{lll}
0 & 1 & 0 \\
1 & 0 & 1 \\
0 & 0 & 0
\end{array}\right], & A_{3}=\left[\begin{array}{ccc}
-1 & 4 & 0 \\
-4 & -1 & 0 \\
0 & 0 & 1
\end{array}\right] .
\end{array}
$$

The averaged model (27) with duty cycles $\left(d_{1}, d_{2}, d_{3}\right)=$ $(0.2,0.7,0.1)$ is given by

$$
\begin{aligned}
\dot{x}_{\mathrm{av}} & =\left[\begin{array}{ccc}
0 & 0 & 0 \\
0 & 0.2 & 0 \\
0 & 0 & 0
\end{array}\right] x_{\mathrm{av}}, \quad x_{\mathrm{av}}(0)=\Pi_{\cap} x_{0} . \\
\Pi_{\cap} & =\left[\begin{array}{lll}
0 & 0 & 0 \\
0 & 1 & 0 \\
0 & 0 & 0
\end{array}\right] .
\end{aligned}
$$

In Figure 2 we show the evolutions of the system for initial value $x_{0}=(0.1,-2,1.5)^{\top}$ and periodicities $p=0.1 \mathrm{~s}$ and $p=0.02 s$, respectively. It can be seen that the switched $D A E$ converge towards the averaged system.

According to the result of the Example 17, we want to prove that under certain assumptions, the trajectories of the switched system converge to those of the averaged system $(27)-(28)$ with $u(t)=0$ when the switching period $p$ tends to zero.

Theorem 18 Consider the regular switched DAE (18) with periodic switching signal $\sigma$ with period $p>0$ given by (5)-(6) and initial condition $x\left(0^{-}\right)=x_{0}$, and consider the averaged model (27)-(28). Assume that $u(t)=0 \forall t$. Denote by $x_{\sigma, p}(t)$ the (in general discontinuous) impulsefree part of the (in general distributional) solution of (18) with $u(t)=0$ and let $x_{\mathrm{av}}(t)$ be the (smooth) solution of $(27)$ with $u(t)=0$. Consider an arbitrary constant 
$\Delta>p$. If (PA) hold $\forall i \in \Sigma$, then

$$
x_{\sigma, p}(t)-x_{\mathrm{av}}(t)=\mathrm{O}(p),
$$

$\forall t \in[p, \Delta]$.

PROOF. The proof proceeds in three steps.

Step 1: We show that (31) holds for $t=t_{1}=p$.

Invoking Remark 14, the impulse-free part of the solution of (18) and the solution of (27) at $t_{1}$ can be written as

$$
\begin{aligned}
x_{\sigma, p}\left(t_{1}^{+}\right) & =\Pi_{1} x_{\sigma, p}\left(t_{1}^{-}\right)=\Pi_{1} H_{\mathrm{diff}}(p) x_{0} \\
x_{\mathrm{av}}\left(t_{1}\right) & =H_{\mathrm{av}}(p) \Pi_{\cap} x_{0} .
\end{aligned}
$$

By taking into account the Taylor approximation (1) together with (21), we have

$$
\begin{aligned}
H_{\mathrm{diff}}(p) & =\Pi_{\cap}+\tilde{A} p+\mathrm{O}\left(p^{2}\right)=\Pi_{\cap}+\mathrm{O}(p) \\
H_{\mathrm{av}}(p) & =I+A_{\text {av }} p+\mathrm{O}\left(p^{2}\right)=I+\mathrm{O}(p),
\end{aligned}
$$

where

$$
\begin{aligned}
\tilde{A}:= & A_{\mathrm{q}}^{\text {diff }} \Pi_{\cap} d_{\mathrm{q}}+\Pi_{\mathrm{q}} A_{\mathrm{q}-1}^{\text {diff }} \Pi_{\mathrm{q}-1} \cdots \Pi_{1} d_{\mathrm{q}-1}+\ldots \\
& +\Pi_{\mathrm{q}} \Pi_{\mathrm{q}-1} \cdots \Pi_{2} A_{2}^{\text {diff }} \Pi_{1} d_{2}+\Pi_{\cap} A_{1}^{\text {diff }} d_{1} .
\end{aligned}
$$

Then

$$
\begin{aligned}
x_{\sigma, p}\left(t_{1}^{+}\right)-x_{\mathrm{av}}\left(t_{1}\right)= & \left(\Pi_{1}\left(\Pi_{\cap}+\mathrm{O}(p)\right)\right. \\
& \left.-(I+\mathrm{O}(p)) \Pi_{\cap}\right) x_{0} \\
= & \left(\Pi_{1} \Pi_{\cap}-\Pi_{\cap}\right) x_{0}+\mathrm{O}(p)=\mathrm{O}(p) .
\end{aligned}
$$

where we used $\Pi_{1} \Pi_{\cap} x_{0}=\Pi_{\cap} x_{0}$ because of (PA.1).

Step 2: We show that (31) holds for any $\left\{t_{k}\right\}_{k=2}^{\ell(p)}$ where $\ell(p)$ is the integer such that $\Delta-p<p \ell(p) \leq \Delta$. Clearly $p \ell(p)=\mathrm{O}(1)$.

By applying the Taylor approximation (1) to the solution of the impulse-free part of the switched system, we have

$$
\begin{aligned}
x_{\sigma, p}\left(t_{k}^{+}\right)= & \Pi_{1} H_{\mathrm{diff}}^{k}(p) x_{0} \\
= & \left(\Pi_{\cap}+\tilde{A} p+\mathrm{O}\left(p^{2}\right)\right)^{k} x_{0} \\
= & \left(\Pi_{\cap}+\mathrm{O}(p)\right)\left(\Pi_{\cap}+\tilde{A} p+\mathrm{O}\left(p^{2}\right)\right)^{k-2}\left(\Pi_{\cap}\right. \\
& +\mathrm{O}(p)) x_{0},
\end{aligned}
$$

for $k=2, \ldots, \ell(p)$. Taking into account (PA.1) together with Lemma 4 , and by applying (3) we obtain

$$
x_{\sigma, p}\left(t_{k}^{+}\right)=\Pi_{\cap}\left(\Pi_{\cap}+\tilde{A} p+\mathrm{O}\left(p^{2}\right)\right)^{k-2} \Pi_{\cap} x_{0}+\mathrm{O}(p) .
$$

Invoking (1) and (3) we can rewrite the solution of the averaged system (29) where $\mathcal{I}_{a v}(p)\left\{u_{k}\right\}=0$ as

$$
x_{\text {av }}\left(t_{k}\right)=\Pi_{\cap}\left(\Pi_{\cap}+A_{\text {av }} p+\mathrm{O}\left(p^{2}\right)\right)^{k-2} \Pi_{\cap} x_{0}+\mathrm{O}(p) .
$$

Hence, invoking $\Pi_{\cap} \tilde{A} \Pi_{\cap}=\Pi_{\cap} A_{\mathrm{av}} \Pi_{\cap}$ and (4), we arrive at

$$
\begin{aligned}
& x_{\sigma, p}\left(t_{k}^{+}\right)-x_{\mathrm{av}}\left(t_{k}\right)=\Pi_{\cap}\left(\left(\Pi_{\cap}+\tilde{A} p+\mathrm{O}\left(p^{2}\right)\right)^{k-2}\right. \\
& \left.-\left(\Pi_{\cap}+A_{\mathrm{av}} p+\mathrm{O}\left(p^{2}\right)\right)^{k-2}\right) \Pi_{\cap} x_{0}+\mathrm{O}(p)=\mathrm{O}(p),
\end{aligned}
$$

for $k=2, \ldots, \ell(p)$.

Step 3: We show that (31) holds for time instants different from multiples of the period $p$.

The solution of (18) and (27) for any $\tau \in\left[s_{k, i}, s_{k, i+1}\right)$ with $i \in \Sigma$ and $k \in \mathbb{N}$, can be written respectively as

$$
\begin{aligned}
x_{\sigma, p}(\tau) & =e^{A_{i}^{\mathrm{diff}}\left(\tau-s_{k, i}\right)} x_{\sigma, p}\left(s_{k, i}^{+}\right) \\
x_{\mathrm{av}}(\tau) & =e^{A_{\mathrm{av}}\left(\tau-s_{k, i}\right)} x_{\mathrm{av}}\left(s_{k, i}\right) .
\end{aligned}
$$

Considering (1) with $s=\tau-s_{k, i}$ we have

$$
x_{\sigma, p}(\tau)-x_{\mathrm{av}}(\tau)=x_{\sigma, p}\left(s_{k, i}^{+}\right)-x_{\mathrm{av}}\left(s_{k, i}\right)+\mathrm{O}(p) .
$$

Taking into account Remark 16 we can write

$$
\begin{aligned}
x_{\sigma, p}\left(s_{k, i}^{+}\right)-x_{\mathrm{av}}\left(s_{k, i}\right) & =\Pi_{i} x_{\sigma, p}\left(s_{k, i}^{-}\right)-x_{\mathrm{av}}\left(s_{k, i}\right) \\
& =\Pi_{i}\left(x_{\sigma, p}\left(s_{k, i}^{-}\right)-x_{\mathrm{av}}\left(s_{k, i}\right)\right) .
\end{aligned}
$$

Then by concatenating (36) for increasing values of $i \in \Sigma$ and $k=1, \ldots, \ell(p)$, and by using (33) and (34) it follows that (31) holds $\forall t \in[p, \Delta]$.

Example 19 The averaged model conjectured in Example 17 can be now confirmed. Indeed the consistency projectors of the switched DAEs of Example 17 are given by

$$
\Pi_{1}=\left[\begin{array}{lll}
0 & 0 & 0 \\
0 & 1 & 0 \\
1 & 0 & 1
\end{array}\right], \quad \Pi_{2}=\left[\begin{array}{lll}
1 & 0 & 0 \\
0 & 1 & 0 \\
0 & 0 & 0
\end{array}\right], \quad \Pi_{3}=\left[\begin{array}{lll}
1 & 0 & 1 \\
0 & 1 & 0 \\
0 & 0 & 0
\end{array}\right],
$$

It is easily to see that (PA) hold for all $\Pi_{i}, i=1, \ldots, 3$. Hence Theorem 18 can be applied and the averaging result holds.

Remark 20 Note that from Lemma \& it follows that commutativity of the consistence projectors, i.e.

$$
\Pi_{i} \Pi_{j}=\Pi_{j} \Pi_{i}, \quad \forall i, j \in \Sigma,
$$




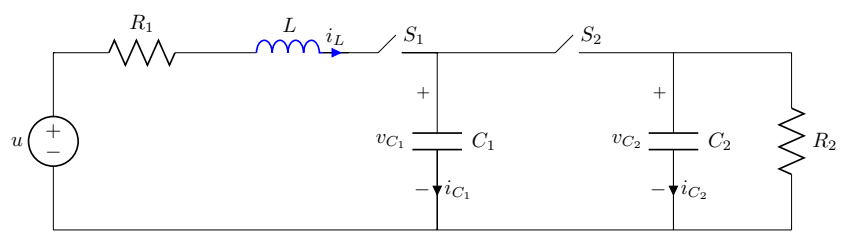

Fig. 3. Electrical circuit with two capacitors and one inductor.

implies (PA.1) and (PA.2), hence our result generalizes the one in [5]. In fact, by analyzing the consistency projectors in Example 19, we can see that they do not commute but (PA) hold, i.e. the commutativity of the consistency projectors is not necessary for averaging.

Remark 21 Theorem 18 makes a statement about the switched DAE (18) where $u(t)=0 \forall t$; however, it is also applicable to switched ODE with jumps of the form (23) with $u(t)=0$. For this it is not necessary that $A_{i}^{\text {diff }}$ and $\Pi_{i}^{\text {diff }}, i \in \Sigma$, are defined in terms of regular matrix pairs $\left(E_{i}, A_{i}\right)$; it suffices that the following properties hold: $\Pi_{i}^{2}=\Pi_{i}, \Pi_{i} A_{i}^{\mathrm{diff}}=A_{i}^{\mathrm{diff}}=A_{i}^{\mathrm{diff}} \Pi_{i}, i \in \Sigma$, i.e. $\Pi_{i}$ must be projectors which are compatible with the corresponding flow matrices $A_{i}^{\mathrm{diff}}$. Then (PA) also ensures convergence towards an averaged system for switched $O D E$ with jumps.

Remark 22 The Projector Assumption means that, differently from the classical averaging result on switched ODEs, the averaging result for swithed DAEs depends on the sequence of modes because of the presence of $\Pi_{\cap}$ in (28). For instance, by considering in Example 17 the sequence of modes 1,3,2 instead of 1,2,3, the condition (PA.1) is no more satisfied and convergence towards the average system does not occur anymore.

\subsection{Non-homogeneous switched DAEs}

In the following we want to analyze the case of a switched non-homogeneous DAE.

Example 23 Consider the switched capacitor circuit shown in Figure 3. By applying the Kirchhoff's laws to the four different configurations obtained by combining the different states of the two ideal switches, the system can be described as a switched DAEs where the state variables are the voltage on the two capacitors and the current through the inductor respectively, $x=\left[v_{C_{1}}, v_{C_{2}}, i_{L}\right]^{\top}$.
Table 1

\begin{tabular}{|c|c|c|}
\hline i-th mode & S1 & S2 \\
\hline 1 & "closed" & "open" \\
2 & "closed" & "closed" \\
3 & "open" & "closed" \\
4 & "open" & "open" \\
\hline
\end{tabular}

Modes of the electrical circuit.

The matrices $\left(E_{i}, A_{i}, B_{i}\right)$ are given by

$$
\begin{aligned}
E_{1}=\left[\begin{array}{ccc}
C_{1} & 0 & 0 \\
0 & C_{2} & 0 \\
0 & 0 & L
\end{array}\right] & A_{1}=\left[\begin{array}{ccc}
0 & 0 & 1 \\
0 & -\frac{1}{R_{2}} & 0 \\
1 & 0 & -R_{1}
\end{array}\right] & B_{1}=\left[\begin{array}{l}
0 \\
0 \\
1
\end{array}\right], \\
E_{2}=\left[\begin{array}{ccc}
C_{1} & C_{2} & 0 \\
0 & 0 & L \\
0 & 0 & 0
\end{array}\right] & A_{2}=\left[\begin{array}{ccc}
0 & -\frac{1}{R_{2}} & 1 \\
-1 & 0 & -R_{1} \\
1 & -1 & 0
\end{array}\right] & B_{2}=\left[\begin{array}{l}
0 \\
1 \\
0
\end{array}\right], \\
E_{3}=\left[\begin{array}{ccc}
C_{1} & C_{2} & 0 \\
0 & 0 & 0 \\
0 & 0 & 0
\end{array}\right] & A_{3}=\left[\begin{array}{ccc}
0-\frac{1}{R_{2}} & 0 \\
1 & -1 & 0 \\
0 & 0 & 1
\end{array}\right] & B_{3}=\left[\begin{array}{l}
0 \\
0 \\
0
\end{array}\right], \\
E_{4}=\left[\begin{array}{ccc}
C_{1} & 0 & 0 \\
0 & C_{2} & 0 \\
0 & 0 & 0
\end{array}\right] & A_{4}=\left[\begin{array}{ccc}
0 & 0 & 0 \\
0 & -\frac{1}{R_{2}} & 0 \\
0 & 0 & 1
\end{array}\right] & B_{4}=\left[\begin{array}{l}
0 \\
0 \\
0
\end{array}\right] .
\end{aligned}
$$

where the $i$-th mode is defined according to the positions of the switches $S_{1}, S_{2}$ as indicated in Table 23.

Consider the following constants:

$$
\rho_{1}=\frac{C_{1}}{C_{1}+C_{2}} \quad \rho_{2}=\frac{C_{2}}{C_{1}+C_{2}},
$$

then the differential projectors are

$$
\begin{aligned}
& \Pi_{1}^{\text {diff }}=\left[\begin{array}{ccc}
1 / C_{1} & 0 & 0 \\
0 & 1 / C_{2} & 0 \\
0 & 0 & 1 / L
\end{array}\right], \quad \Pi_{2}^{\text {diff }}=\left[\begin{array}{ccc}
\frac{\rho_{1}}{C_{1}} & 0 & -\frac{\rho_{1}^{2}}{R_{2} C_{1}} \\
\frac{\rho_{1}}{C_{1}} & 0 & -\frac{\rho_{1}^{2}}{R_{2} C_{1}} \\
0 & 1 / L & \rho_{2}
\end{array}\right], \\
& \Pi_{3}^{\text {diff }}=\left[\begin{array}{ccc}
\frac{\rho_{1}}{C_{1}}-\frac{\rho_{1}^{2}}{R_{2} C_{1}} & 0 \\
\frac{\rho_{1}}{C_{1}}-\frac{\rho_{1}^{2}}{R_{2} C_{1}} & 0 \\
0 & 0 & 0
\end{array}\right], \quad \Pi_{4}^{\text {diff }}=\left[\begin{array}{ccc}
1 / C_{1} & 0 & 0 \\
0 & 1 / C_{2} & 0 \\
0 & 0 & 0
\end{array}\right] \text {. }
\end{aligned}
$$

The candidate averaged model (27)-(28) is given by the following

$$
\begin{aligned}
\dot{x}_{a v}(t) & =A_{a v} x_{a v}(t)+B_{a v} u(t) \quad x_{\mathrm{av}}(0)=\Pi_{\cap} x_{0}, \\
A_{a v} & =\left[\begin{array}{ccc}
-\frac{\rho_{1}^{2}}{R_{2} C_{1}} & -\frac{\rho_{2}^{2}}{R_{2} C_{1}} & 0 \\
-\frac{\rho_{1}^{2}}{R_{2} C_{1}} & -\frac{\rho_{2}^{2}}{R_{2} C_{1}} & 0 \\
0 & 0 & 0
\end{array}\right], \quad B_{a v}=0, \\
\Pi_{\cap} & =\left[\begin{array}{ccc}
\rho_{1} & \rho_{2} & 0 \\
\rho_{1} & \rho_{2} & 0 \\
0 & 0 & 0
\end{array}\right] .
\end{aligned}
$$

For the simulations the following parameters have been used: $C_{1}=80.36 \mathrm{mF}, C_{2}=8.2 \mathrm{mF}, L=5 \mathrm{H}, R_{2}=$ $20 \Omega, \quad R_{1}=10 \Omega$ and $\bar{u}=5 \mathrm{~V}$ with duty cycles $\left(d_{1}, d_{2}, d_{3}, d_{4}\right)=(0.3,0.4,0.2,0.1)$ and initial conditions $x_{0}=(1,1,0)^{\top}$. The evolutions of the state variables are shown in Figure 4. We can see that by decreasing the switching period from $p=0.1$ s to $p=0.02 s$ 

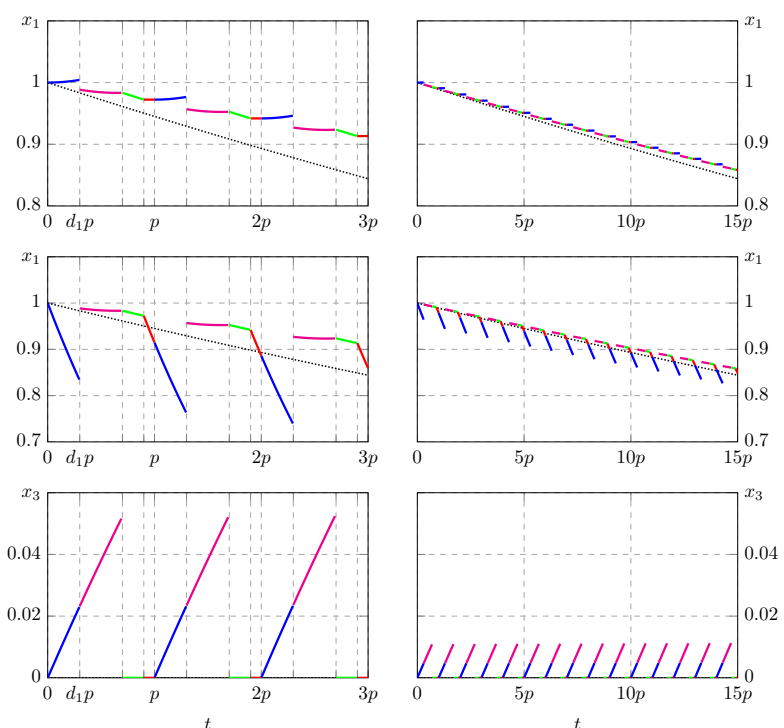

Fig. 4. Evolution of the state variables (first component top, second component middle, third component bottom)for slow switching ( $p=0.1 s$, left) and fast switching ( $p=0.02 s$, right). The averaging dynamics are plotted with dotted black lines, while the trajectories of the switched DAE are colored according to the active mode (mode 1 blue, mode 2 magenta, mode 3 green, mode 4 red).

the error between the averaged evolutions and the ones of the switched system reduces with order $p$.

According to the result of Example 23, the following theorem gives the conditions that must be satisfied to extend the averaging result for the switched DAEs (18) with nonzero inputs.

Theorem 24 Consider the regular switched DAE (18) with periodic switching signal $\sigma$ with period $p>0$ given by (5)-(6) and initial condition $x\left(0^{-}\right)=x_{0}$, and consider the averaged model (27)-(28). Denote by $x_{\sigma, p}(t)$ the (in general discontinuous) impulse-free part of the (in general distributional) solution of (18) and let $x_{\mathrm{av}}(t)$ be the (smooth) solution of (27). Consider an arbitrary constant $\Delta>p$. If (PA) and (22) hold $\forall i \in \Sigma$ and the input $u: \mathbb{R}_{+} \rightarrow \mathbb{R}^{m}$ is Lipschitz continuous with constant $L$, then

$\forall t \in[p, \Delta]$

$$
x_{\sigma, p}(t)-x_{\mathrm{av}}(t)=\mathrm{O}(p)
$$

For the proof of Theorem 24 the following Lemma is needed.

Lemma 25 Consider the functions (26b) and (30b). Assume that (PA.2) holds and that the input $u: \mathbb{R}_{+} \rightarrow$ $\mathbb{R}^{m}$ is Lipschitz continuous with constant $L$. Then

$$
\Pi_{\cap} \mathcal{I}_{\text {diff }}(p)\left\{u_{j}\right\}-\mathcal{I}_{\text {av }}(p)\left\{u_{j}\right\}=\mathrm{O}\left(p^{2}\right),
$$

for $j=1, \ldots, \ell(p)-1$.

PROOF. Applying the Taylor approximation of the exponential matrix (1) to (26b) and (30b) we obtain

$$
\begin{aligned}
\mathcal{I}_{\text {diff }}(p)\left\{u_{j}\right\}= & \sum_{i=1}^{\mathrm{q}}\left[\left(\Pi_{\mathrm{q}} \Pi_{\mathrm{q}-1} \cdots \Pi_{i+1}+\mathrm{O}(p)\right) \times\right. \\
& \left.\times \int_{c_{i-1}}^{c_{i}}(I+\mathrm{O}(p)) B_{i}^{\text {diff }} u_{j}(\xi) \mathrm{d} \xi\right] \\
\mathcal{I}_{\mathrm{av}}(p)\left\{u_{j}\right\}= & \int_{0}^{p}(I+\mathrm{O}(p-\xi)) B_{\mathrm{av}} u_{j}(\xi) \mathrm{d} \xi
\end{aligned}
$$

where we used that $\mathrm{O}\left(c_{i}-\xi\right)$ can be substituted by $\mathrm{O}(p)$ since $\left(c_{i}-\xi\right) \leq p, \forall i \in \Sigma$.

Furthermore taking into account that $\frac{1}{b-a} \int_{a}^{b} f(t) \mathrm{d} t=$ $f(\alpha)$, with $\alpha \in[a, b]$ we have

$$
\begin{aligned}
& \mathcal{I}_{\text {diff }}(p)\left\{u_{j}\right\}=\sum_{i=1}^{\mathrm{q}} \Pi_{\mathrm{q}} \cdots \Pi_{i+1} B_{i}^{\text {diff }} u_{j}\left(\alpha_{i}\right) d_{i} p+\mathrm{O}\left(p^{2}\right) \\
& \mathcal{I}_{\text {av }}(p)\left\{u_{j}\right\}=B_{\text {av }} u_{j}\left(\alpha_{\mathrm{q}+1}\right) p+\mathrm{O}\left(p^{2}\right)
\end{aligned}
$$

where $\alpha_{i} \in\left[c_{i-1}, c_{i}\right]$ and $\alpha_{\mathrm{q}+1} \in[0, p]$.

Due to (PA.2) we have that

$$
\Pi_{\cap} \mathcal{I}_{\text {diff }}(p)\left\{u_{j}\right\}=\sum_{i=1}^{\mathrm{q}} \Pi_{\cap} B_{i}^{\mathrm{diff}} u_{j}\left(\alpha_{i}\right) d_{i} p+\mathrm{O}\left(p^{2}\right)
$$

Hence, considering

$$
\begin{aligned}
& \left\|\left(\sum_{i=1}^{\mathrm{q}} \Pi_{\cap} B_{i}^{\text {diff }} u_{j}\left(\alpha_{i}\right) d_{i} p\right)-B_{a v} u_{j}\left(\alpha_{\mathrm{q}+1}\right) p\right\| \\
& \leq \sum_{i=1}^{\mathrm{q}}\left\|\Pi_{\cap} B_{i}^{\text {diff }} u_{j}\left(\alpha_{i}\right) d_{i} p-\Pi_{\cap} B_{i}^{\text {diff }} u_{j}\left(\alpha_{\mathrm{q}+1}\right) d_{i} p\right\| \\
& \leq \sum_{i=1}^{\mathrm{q}}\left\|\Pi_{\cap} B_{i}^{\text {diff }}\right\| L\left\|\alpha_{i}-\alpha_{\mathrm{q}+1}\right\| d_{i} p \\
& \leq \sum_{i=1}^{\mathrm{q}}\left\|\Pi_{\cap} B_{i}^{\text {diff }}\right\| L d_{i} p^{2},
\end{aligned}
$$

and by combining the last inequality with (43) and (44) we obtain that (41) holds.

Proof of Theorem 24 The proof proceeds in two steps.

Step 1: We show that (40) holds for any $\left\{t_{k}\right\}_{k=1}^{\ell(p)}$ where $\ell(p)$ is an integer such that $\Delta-p<\ell(p) p \leq \Delta$ where $p \ell(p)=\mathrm{O}(1)$. 
The solutions of (23) and (27) are given by (25) and (29), respectively.

Due to assumption (PA) the averaging result (31) for the homogeneous part holds, hence

$$
H_{\mathrm{diff}}(p)^{k} x_{0}-H_{\mathrm{av}}(p)^{k} \Pi_{\cap} x_{0}=\mathrm{O}(p)
$$

$\forall k=1, \ldots, \ell(p)$.

By considering (32), taking into account (3) and (PA.1) and noting that $\mathcal{I}_{\text {diff }}(p)\left\{u_{i}\right\}$ and $\mathcal{I}_{\text {av }}(p)\left\{u_{i}\right\}$ are $\mathrm{O}(p)$ functions we obtain

$$
\begin{aligned}
x_{\sigma, p}\left(t_{k}\right)-x_{\mathrm{av}}\left(t_{k}\right) & =\sum_{i=0}^{k-2}\left(H_{\mathrm{diff}}(p)^{k-1-i} \Pi_{\cap} \mathcal{I}_{\mathrm{diff}}(p)\left\{u_{i}\right\}\right. \\
& \left.-H_{\mathrm{av}}(p)^{k-1-i} \mathcal{I}_{\mathrm{av}}(p)\left\{u_{i}\right\}\right)+\mathrm{O}(p)
\end{aligned}
$$

Consider a generic $j$-th term of $(46)$, for $j=1, \ldots, \ell(p)-$ 1 then we have

$$
\begin{aligned}
& H_{\mathrm{diff}}(p)^{j} \Pi_{\cap} \mathcal{I}_{\mathrm{diff}}(p)\left\{u_{j}\right\}-H_{\mathrm{av}}(p)^{j} \mathcal{I}_{\mathrm{av}}(p)\left\{u_{j}\right\}= \\
& H_{\mathrm{diff}}(p)^{j} \Pi_{\cap} \mathcal{I}_{\mathrm{diff}}(p)\left\{u_{j}\right\}-H_{\mathrm{av}}(p)^{j} \Pi_{\cap} \mathcal{I}_{\mathrm{diff}}(p)\left\{u_{j}\right\} \\
&+H_{\mathrm{av}}(p)^{j} \Pi_{\cap} \mathcal{I}_{\mathrm{diff}}(p)\left\{u_{j}\right\}-H_{\mathrm{av}}(p)^{j} \mathcal{I}_{\mathrm{av}}(p)\left\{u_{j}\right\} \\
&=\left(H_{\mathrm{diff}}(p)^{j}-H_{\mathrm{av}}(p)^{j} \Pi_{\cap}\right) \Pi_{\cap} \mathcal{I}_{\mathrm{diff}}(p)\left\{u_{j}\right\} \\
&+H_{\mathrm{av}}(p)^{j}\left(\Pi_{\cap} \mathcal{I}_{\mathrm{diff}}(p)\left\{u_{j}\right\}-\mathcal{I}_{\mathrm{av}}(p)\left\{u_{j}\right\}\right) \\
&= \mathrm{O}\left(p^{2}\right)
\end{aligned}
$$

where we used (45), Lemma 25 and (3).

Then by using (46), we have that

$x_{\sigma, p}\left(t_{k}^{+}\right)-x_{\mathrm{av}}\left(t_{k}\right)=(k-2) \mathrm{O}\left(p^{2}\right)+\mathrm{O}(p)=\mathrm{O}(p) \forall\left\{t_{k}\right\}_{k=1}^{\ell(p)}$.

Step 2: We show that (40) holds for all time instants different from multiples of $p$.

The solutions of the switched and averaged system evaluated in $\tau \in\left[s_{k, i}, s_{k, i+1}\right)$, with $i \in \mathbb{N}$ and $k \in \mathbb{N}$, can be written in the form (35). Hence by applying the Taylor expression (1) we obtain (36). Then considering (37) and (47), the proof follows by concatenating (36) for increasing $i \in \Sigma$ and $k=1, \ldots, \ell(p)+1$.

Example 26 The consistency projectors of Example 23 are given by

$$
\begin{array}{rlrl}
\Pi_{1} & =\left[\begin{array}{lll}
1 & 0 & 0 \\
0 & 1 & 0 \\
0 & 0 & 1
\end{array}\right], & \Pi_{2} & =\left[\begin{array}{ccc}
\rho_{1} & \rho_{2} & 0 \\
\rho_{1} & \rho_{2} & 0 \\
0 & 0 & 1
\end{array}\right], \\
\Pi_{3} & =\left[\begin{array}{lll}
\rho_{1} & \rho_{2} & 0 \\
\rho_{1} & \rho_{2} & 0 \\
0 & 0 & 0
\end{array}\right], & \Pi_{4}=\left[\begin{array}{lll}
1 & 0 & 0 \\
0 & 1 & 0 \\
0 & 0 & 0
\end{array}\right] .
\end{array}
$$

We can see that the projector assumptions (PA) hold. Furthermore by analyzing the following impulsive projec- tors

$$
\begin{array}{rlrl}
\Pi_{1}^{\mathrm{imp}} & =\left[\begin{array}{lll}
0 & 0 & 0 \\
0 & 0 & 0 \\
0 & 0 & 0
\end{array}\right], & \Pi_{2}^{\mathrm{imp}}=\left[\begin{array}{ccc}
0 & 0 & \rho_{2} \\
0 & 0 & -\rho_{1} \\
0 & 0 & 0
\end{array}\right], \\
\Pi_{3}^{\mathrm{imp}}=\left[\begin{array}{ccc}
0 & \rho_{2} & 0 \\
0 & -\rho_{1} & 0 \\
0 & 0 & 1
\end{array}\right], & \Pi_{4}^{\mathrm{imp}}=\left[\begin{array}{lll}
0 & 0 & 0 \\
0 & 0 & 0 \\
0 & 0 & 1
\end{array}\right],
\end{array}
$$

and the $B_{i}$ matrices with $i=1, \ldots, 4$ we see that $(22)$ is also satisfied. Hence, according to Theorem 24, the averaging result holds as confirmed by the simulations in Figure 4.

The Lipschitz assumption on the input $u$ introduced in Lemma 25 and used for the proof of Theorem 24, can be relaxed in a particular case as shown by the following result.

Proposition 27 Consider a non-homogeneous switched $D A E$ where (22) and the averaging result for the corresponding homogeneous system hold. Then if the following conditions hold

$$
B_{i}^{\text {diff }}=B_{h}^{\text {diff }}, \quad \forall i, h \in \Sigma
$$

the averaging result (40) is satisfied.

PROOF. By using (42) and noting that the functions $\mathcal{I}_{\text {diff }}(p)\left\{u_{j}\right\}$ and $\mathcal{I}_{\text {av }}(p)\left\{u_{j}\right\}$ are $\mathrm{O}(p)$ we have

$$
\begin{aligned}
& \Pi_{\cap} \mathcal{I}_{\text {diff }}(p)\left\{u_{j}\right\}-\mathcal{I}_{\mathrm{av}}(p)\left\{u_{j}\right\} \\
& =\sum_{i=0}^{\mathrm{q}} \int_{c_{i}}^{c_{i+1}} \Pi_{\cap} B_{i}^{\mathrm{diff}} u_{j}(\xi) \mathrm{d} \xi-\int_{0}^{p} B_{\mathrm{av}} u_{j}(\xi) \mathrm{d} \xi \\
& +\mathrm{O}\left(p^{2}\right) \\
& =\sum_{i=0}^{\mathrm{q}} \int_{c_{i}}^{c_{i+1}} \Pi_{\cap}\left(B_{i}^{\mathrm{diff}}-B_{\mathrm{av}}\right) u_{j}(\xi) \mathrm{d} \xi+\mathrm{O}\left(p^{2}\right),
\end{aligned}
$$

where $j=1, \ldots, \ell(p)-1$. Then considering that

$$
\begin{aligned}
\Pi_{\cap}\left(B_{i}^{\text {diff }}-B_{i}^{\text {diff }} d_{i}\right. & \left.-\sum_{h \neq i \in \Sigma} B_{h}^{\text {diff }} d_{h}\right) \\
& =\Pi_{\cap} \sum_{h \neq i \in \Sigma}\left(B_{i}^{\text {diff }}-B_{h}^{\text {diff }}\right) d_{h},
\end{aligned}
$$

where we use $d_{i}=1-\sum_{h \neq i \in \Sigma} d_{h}$ with $i \in \Sigma$. By combining (49) and (50) with (46) and taking into account (31), the averaging result (40) holds for any $\left\{t_{k}\right\}_{k=1}^{\ell(p)}$. It is easy to prove that (40) holds for all time instants different from multiples of $p$, then the proof is complete.

Remark 28 In the case of a switched DAE with two modes, the averaging results proved in Theorem 18, Theorem 24 and Proposition 27, hold even if $\Pi_{\cap}$ is a projector but assumption (PA.2) doesn't hold. 


\section{$4 \quad$ Stability via fast switching}

The averaging result in Theorem 18 can be used for the stability analysis of the homogeneous switched DAE

$$
E_{\sigma(t)} \dot{x}(t)=A_{\sigma(t)} x(t), \quad t \in \mathbb{R}_{+},
$$

with $\sigma$ given by (5)-(6).

Remark 29 The proof of Theorem 18 reveals that the constant involved in the big-O bound depends linearly on $\left\|x_{0}\right\|$ and $\Delta$. Then by considering (34) we have that

$$
\left\|x_{\sigma, p}(t)-x_{\mathrm{av}}(t)\right\| \leq c\left\|x_{0}\right\| \Delta p, \quad \forall t \in[p, \Delta]
$$

for sufficiently small $p$ and a constant $c>0$ independent of $p, x_{0}$ and $\Delta$.

Theorem 30 Consider the regular switched DAE (51) with periodic switching signal $\sigma$ given by (5)-(6) and initial condition $x\left(0^{-}\right)=x_{0}$, and consider the averaged model (27)-(28). Denote by $x_{\sigma, p}(t)$ the (in general discontinuous) impulse-free part of the (in general distributional) solution of (51) and let $x_{\mathrm{av}}(t)$ be the (smooth) solution of (27) with $u(t)=0 \forall t$. If the averaged system (27) is exponentially stable, then there exists a switching period $p$, say $p^{*}$, such that the switched system (51) is exponentially stable.

PROOF. Due to the exponential stability of the averaged system we can choose a fixed time instant $T>0$, such that

$$
\left\|x_{\mathrm{av}}(T)\right\| \leq \frac{1}{2}\left\|x_{\mathrm{av}}(T / 2)\right\|
$$

for all initial conditions $x_{0} \in \mathbb{R}^{n}$ in (27). Let

$$
c:=\min \left\{\left\|e^{A_{\mathrm{av}} T / 2} \Pi_{\cap} x_{0}\right\| \mid\left\|\Pi_{\cap} x_{0}\right\|=1\right\}>0,
$$

where positivity follows from the continuity of the map $z \mapsto e^{A_{\text {av }} T / 2} z$ and triviality of the kernel of the matrix $e^{A_{\text {av } T / 2}}$. Because of (31) we can choose $\bar{p}>0$ sufficiently small such that

$$
\begin{aligned}
\left\|x_{\mathrm{av}}(T)-x_{\sigma, p}\left(T^{-}\right)\right\| & \leq \frac{c}{8} \leq \frac{1}{8}\left\|x_{\mathrm{av}}(T / 2)\right\| \\
\left\|x_{\sigma, p}\left(T / 2^{-}\right)-x_{\mathrm{av}}(T / 2)\right\| & \leq \frac{c}{8} \leq \frac{1}{8}\left\|x_{\mathrm{av}}(T / 2)\right\|
\end{aligned}
$$

$\forall p \in(0, \bar{p})$ and all solutions of (51) and (31) where we consider, without loss of generality, initial conditions $x_{0}$ satisfying $\left\|\Pi_{\cap} x_{0}\right\|=1$.
Combining (53) with (52), and by using the reverse triangle inequality, we obtain

$$
\begin{aligned}
\left\|x_{\sigma, p}\left(T^{-}\right)\right\| & \leq\left\|x_{\mathrm{av}}(T)\right\|+\frac{1}{8}\left\|x_{\mathrm{av}}(T / 2)\right\| \\
& \leq \frac{1}{2}\left\|x_{\mathrm{av}}(T / 2)\right\|+\frac{1}{8}\left\|x_{\mathrm{av}}(T / 2)\right\| \\
& =\frac{5}{8}\left\|x_{\mathrm{av}}(T / 2)\right\|
\end{aligned}
$$

and (54) together with the reverse triangle inequality, implies

$$
\begin{aligned}
\left\|x_{\sigma, p}\left(T / 2^{-}\right)\right\| & \geq \mid\left\|x_{\mathrm{av}}(T / 2)\right\|-\frac{1}{8}\left\|x_{\mathrm{av}}(T / 2)\right\| \\
& \geq \frac{7}{8}\left\|x_{\mathrm{av}}(T / 2)\right\| .
\end{aligned}
$$

Altogether, we arrive at

$$
\left\|x_{\sigma, p}\left(T^{-}\right)\right\| \leq \frac{5}{7}\left\|x_{\sigma, p}\left(T / 2^{-}\right)\right\|
$$

i.e. we have shown that for all initial conditions there is a reduction of at least $5 / 7$ of the norm of the state on a time interval of length $T / 2$ and for all sufficiently small switching periods $p$. Without restriction, we can choose a $p^{*}=T /(2 \theta)$ for sufficiently large $\theta \in \mathbb{N}$. Consider the solution of (51) as a concatenation of transition matrices defined as

$$
\Phi_{p^{*}, i}:=e^{A_{i}^{\text {diff }} d_{i} p^{*}} \Pi_{i}
$$

then let us introduce for $t_{1}>t_{0} \geq 0$ the state transition matrix $\Phi_{\sigma, p}^{t_{0}^{-} \rightarrow t_{1}^{-}} \in \mathbb{R}^{n \times n}$ which maps any (possibly inconsistent) initial value $x_{0} \in \mathbb{R}^{n}$ at $t_{0}^{-}$to the value of $x\left(t_{1}^{-}\right)$, in particular,

$$
x_{\sigma, p^{*}}\left(t_{1}^{+}\right)=\Phi_{\sigma, p^{*}}^{t_{0} \rightarrow t_{1}} x_{\sigma, p^{*}}\left(t_{0}^{-}\right),
$$

for all solutions of (51) and all $t_{1}>t_{0} \geq 0$. From (57) it follows that

$$
\left\|\Phi_{\sigma, p^{*}}^{T / 2 \rightarrow T}\right\| \leq 5 / 7 \text {. }
$$

From $T / 2=\theta p^{*}$ for $\theta \in \mathbb{N}$ and the periodicity of the switching signal it follows that

$$
\Phi_{\sigma, p^{*}}^{k T / 2 \rightarrow(k+1) T / 2}=\Phi_{\sigma, p^{*}}^{T / 2 \rightarrow T} \quad \forall k \in \mathbb{N} \backslash\{0\},
$$

in particular, by considering that $T / 2$ is a multiple of the switching period $p^{*}$,

$$
x_{\sigma, p^{*}}\left(k T / 2^{-}\right)=\left(\Phi_{\sigma, p^{*}}^{T / 2 \rightarrow T}\right)^{k-1} \Phi_{\sigma, p^{*}}^{0 \rightarrow T / 2} x_{0}
$$

and hence

$$
\left\|x_{\sigma, p^{*}}\left(k T / 2^{-}\right)\right\| \leq\left(\frac{5}{7}\right)^{k-1}\left\|\Phi_{\sigma, p^{*}}^{0 \rightarrow T / 2}\right\|\left\|x_{0}\right\| .
$$


From (54), by applying the reverse triangle inequality we have that

$$
\left\|\Phi_{\sigma, p}^{0 \rightarrow T / 2}\right\|\left\|x_{0}\right\| \leq\left\|x_{\mathrm{av}}(T / 2)\right\|+\alpha p .
$$

with a suitable constant $\alpha>0$. Hence, considering (1) we can conclude that

$$
\Phi_{\sigma, p}^{0 \rightarrow T / 2}=e^{A_{\text {av }} T / 2}+\mathrm{O}(p)=\mathrm{O}(1)
$$

Furthermore, for $\tau \in(0, T / 2)$ we have that

$$
x_{\sigma, p^{*}}\left(k T / 2+\tau^{-}\right)=\Phi_{\sigma, p^{*}}^{T / 2 \rightarrow T / 2+\tau} x_{\sigma, p^{*}}\left(k T / 2^{-}\right)
$$

where

$$
\Phi_{\sigma, p}^{T / 2 \rightarrow T / 2+\tau}=e^{A_{\mathrm{av}} \tau}+\mathrm{O}(p)=\mathrm{O}(1)
$$

Considering the time instant $t=k T / 2+\tau$ and combining (58) with (60), we have that

$$
\left\|x_{\sigma, p^{*}}\left(t^{-}\right)\right\| \leq\left(\frac{5}{7}\right)^{k-1}\left\|\Phi_{\sigma, p^{*}}^{T / 2 \rightarrow T / 2+\tau}\right\|\left\|\Phi_{\sigma, p^{*}}^{0 \rightarrow T / 2}\right\|\left\|x_{0}\right\|
$$

By considering (59), (61), $k=2(t-\tau) / T$ and $\mu=\left(\frac{5}{7}\right)^{2}$ where $\mu \in(0,1)$, we have that for sufficiently small $p^{*}=T /(2 \theta)$, there exists constant $C>0$ such that $\forall$ $t>0$

$$
\left\|x_{\sigma, p^{*}}\left(t^{-}\right)\right\| \leq C \mu^{\lfloor t / T\rfloor}\left\|x_{0}\right\|
$$

which implies exponential stability of the switched system (51).

\section{$5 \quad$ Partial averaging}

The averaging result in Theorem 24 allows to approximate a switched DAE by means of a smooth averaged system. If conditions (PA) are not satisfied, it might be possible to partition the state variable such that the averaging result holds only for a part of the state. The resulting partial averaged model is still a switched system but simpler than the original one. The following example motivates the partial averaging analysis.

Example 31 Consider the electrical circuit of Figure 3 where, for simplicity, the inductor is not considered. Assume as state variables the currents and the voltages of the two capacitors. We can model the system considering the input $u(t)$ as a state variable (note that the state equation that represents the input is given by $d u / d t=0)$. Then the system can be written in the form (51) where the state variables are given by $x=\left[v_{C_{1}}, v_{C_{2}}, u, i_{C_{1}}, i_{C_{2}}\right]^{\top}$.
The E matrix is the same for all modes

$$
E_{i}=\left[\begin{array}{ccccc}
C_{1} & 0 & 0 & 0 & 0 \\
0 & C_{2} & 0 & 0 & 0 \\
0 & 0 & 1 & 0 & 0 \\
0 & 0 & 0 & 0 & 0 \\
0 & 0 & 0 & 0 & 0
\end{array}\right] \quad i=1, \ldots, 4
$$

while the $A_{i}$-matrices $i=1, \ldots, 4$, are given by

$$
\begin{aligned}
A_{1} & =\left[\begin{array}{ccccc}
0 & 0 & 0 & 1 & 0 \\
0 & 0 & 0 & 0 & 1 \\
0 & 0 & 0 & 0 & 1 \\
1 & 0 & -1 & R_{1} & 0 \\
0 & 1 & 0 & 0 & -R_{2}
\end{array}\right], & A_{2}=\left[\begin{array}{ccccc}
0 & 0 & 0 & 1 & 0 \\
0 & 0 & 0 & 0 \\
0 & 0 & 0 & 0 & 0 \\
1 & -1 & 0 & 0 & 0 \\
1 & R_{1} & -1 & R_{1} & R_{1}
\end{array}\right], \\
A_{3} & =\left[\begin{array}{ccccc}
0 & 0 & 0 & 0 & 0 \\
0 & 0 & 0 & 0 & 1 \\
0 & 0 & 0 & 0 & 0 \\
1 & -1 & 0 & 0 & 0 \\
0 & \frac{1}{R_{2}} & 0 & 1 & 1
\end{array}\right], & A_{4}=\left[\begin{array}{ccccc}
0 & 0 & 0 & 1 & 0 \\
0 & 0 & 0 & 0 & 1 \\
0 & 0 & 0 & 0 & 0 \\
0 & 0 & 0 & 1 & 0 \\
0 & 1 & 0 & 0 & -R_{2}
\end{array}\right] .
\end{aligned}
$$

Consider the constants $\rho_{1}$ and $\rho_{2}$ defined in (39) and the following constant $\rho_{3}=\frac{\rho_{1} \rho_{2}\left(R_{1}+R_{2}\right)}{R_{1} R_{2}}$, then the consistency projectors are

$$
\begin{aligned}
\Pi_{1}= & {\left[\begin{array}{ccccc}
1 & 0 & 0 & 0 & 0 \\
0 & 1 & 0 & 0 & 0 \\
0 & 0 & 1 & 0 & 0 \\
-\frac{1}{R_{1}} & 0 & \frac{1}{R_{1}} & 0 & 0 \\
0 & \frac{1}{R_{2}} & 0 & 0 & 0
\end{array}\right], } \\
\Pi_{3}= & {\left[\begin{array}{cccccccc}
\rho_{1} & \rho_{2} & 0 & 0 & 0 \\
\rho_{1} & \rho_{2} & 0 & 0 & 0 \\
0 & 0 & 1 & 0 & 0 \\
-\frac{\rho_{1}^{2}}{R_{2}} & -\frac{\rho_{1} \rho_{2}}{R_{2}} & 0 & 0 & 0 \\
-\frac{\rho_{1} \rho_{2}}{R_{2}} & -\frac{\rho_{2}^{2}}{R_{2}} & 0 & 0 & 0
\end{array}\right], \quad \Pi_{2}=\left[\begin{array}{cccccc}
\rho_{1} & \rho_{2} & 0 & 0 & 0 \\
\rho_{1} & \rho_{2} & 0 & 0 & 0 \\
0 & 0 & 1 & 0 & 0 \\
-\frac{\rho_{3} C_{1}}{C_{2}} & -\rho_{3} & \frac{\rho_{1}}{R_{1}} & 0 & 0 \\
-\rho_{3} & -\frac{\rho_{3} C_{2}}{C_{1}} & \frac{\rho_{2}}{R_{1}} & 0 & 0
\end{array}\right], }
\end{aligned}
$$

we can see that (PA) do not hold and an averaging result as stated in Theorem 18 doesn't hold. The evolutions of the state variables are reported in Figure 5 with different switching periods. The state variables $x_{4}$ and $x_{5}$ present state jumps whose amplitudes are independent from the switching period while the first two state variables seem to converge to an averaging evolution. Note that the simulations are obtained with initial conditions $x_{0}=(1,1,5,0,0)^{\top}$, while the input $x_{3}$ is a constant value given by $x_{0_{3}}=5 \mathrm{~V}$.

From the Example 31 it is clear that in some cases the averaged model is not sufficient to represent the dynamics of the system; there are states jumps whose amplitudes are independent from the switching period and cannot be presented with an averaged model. However it is of interest to analyze a simplified model in which some states are represented by continuous values while for the remaining states some switching is still needed.

We want to find conditions such that a partial averaged model is given by

$$
\begin{aligned}
& \dot{x}_{\mathrm{pav}}(t)=A_{\mathrm{pav}_{i}}^{\mathrm{diff}} x_{\mathrm{pav}}(t)+B_{\mathrm{pav}_{i}}^{\mathrm{diff}} u(t), t \in\left(s_{k, i}, s_{k, i+1}\right) \\
& x_{\mathrm{pav}}\left(s_{k, i}^{+}\right)=\Pi_{i}^{*} x_{\mathrm{pav}}\left(s_{k, i}^{-}\right), \\
& x_{\text {pav }}(0-)=\Pi_{\cap}^{*} x_{0} \text {, }
\end{aligned}
$$



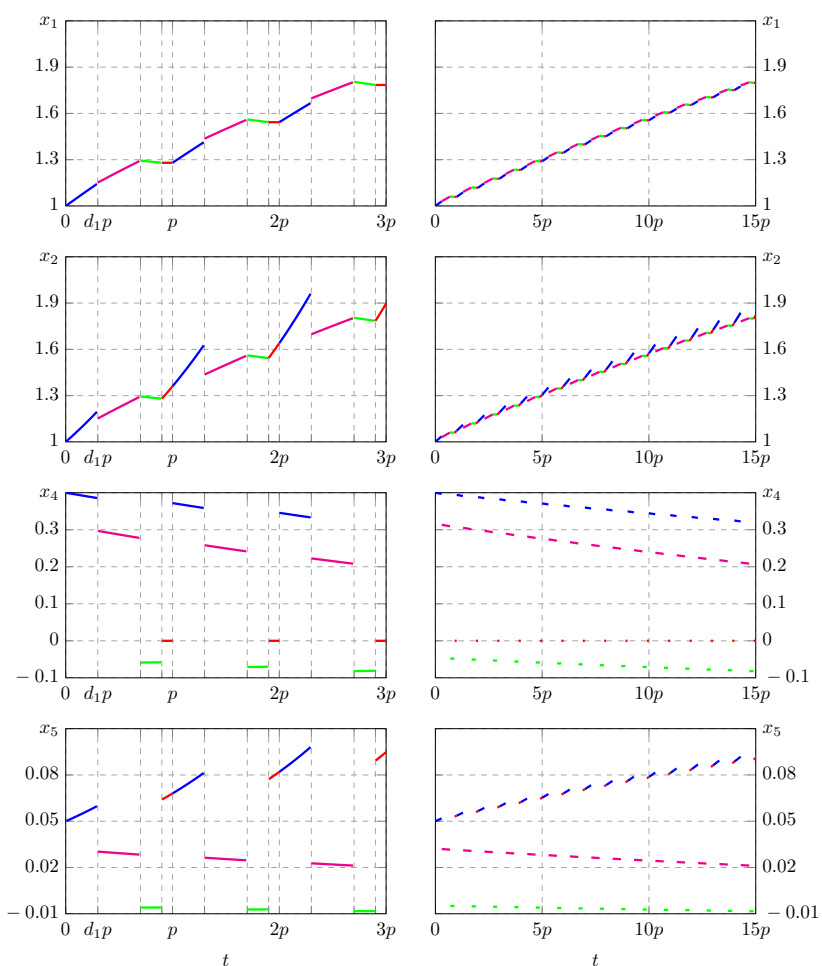

Fig. 5. Evolution of the state variables (first component top, fifth component bottom) for slow switching ( $p=0.1 \mathrm{~s}$, left) and fast switching $(p=0.02 s$, right). The trajectories of the switched DAE are colored according to the active mode (mode 1 blue, mode 2 magenta, mode 3 green, mode 4 red).

where the switching times are given by (5) and

$$
\left.\begin{array}{rl}
A_{\mathrm{pav}_{i}}^{\mathrm{diff}} & :=\left[\begin{array}{cc}
A_{\mathrm{pav}} & 0 \\
A_{21_{i}}^{\mathrm{diff}} & A_{22_{i}}^{\text {diff }}
\end{array}\right] \\
B_{\mathrm{pav}_{i}}^{\mathrm{diff}} & :=\left[\begin{array}{c}
B_{\mathrm{pav}} \\
B_{n-\alpha_{i}}^{\mathrm{diff}}
\end{array}\right] \\
\Pi_{i}^{*} & :=\left[\begin{array}{cc}
I_{\alpha} & 0_{[\alpha \times(n-\alpha)]} \\
\Pi_{21_{i}} & \Pi_{22_{i}}
\end{array}\right] \\
\Pi_{\cap}^{*} & :=\left[\begin{array}{cc}
\Pi_{\cap_{P}} & 0 \\
\Pi_{21_{1}} & \Pi_{22_{1}}
\end{array}\right]
\end{array}\right\},
$$

with

$$
\left.\begin{array}{l}
A_{\text {pav }}:=\Pi_{\cap_{P}} \sum_{i=1}^{\mathrm{q}} d_{i} A_{11_{i}}^{\mathrm{diff}} \Pi_{\cap_{p}} \\
B_{\mathrm{pav}}:=\Pi_{\cap_{P}} \sum_{i=1}^{\mathrm{q}} d_{i} B_{\alpha_{i}}^{\mathrm{diff}}
\end{array}\right\},
$$

where $d_{i}, i \in \Sigma$ is the duty cycle of the $i$-th mode defined by (5) and $\Pi_{\cap_{P}}=\prod_{i=1}^{\mathrm{q}} \Pi_{11_{i}}$. Note that the consistency projectors, the flow matrices and the $B_{i}^{\text {diff }}$ have the following structures

$$
\begin{aligned}
B_{i}^{\text {diff }} & =\left[B_{\alpha_{i}}^{\text {diff }} B_{n-\alpha_{i}}^{\text {diff }}\right]^{\top}, \\
A_{i}^{\text {diff }} & =\left[\begin{array}{cc}
A_{11_{i}[\alpha \times \alpha]}^{\text {diff }} & 0_{[\alpha \times(n-\alpha)]} \\
A_{21_{i[(n-\alpha) \times \alpha]}^{\text {diff }}} & A_{22_{i}[(n-\alpha) \times(n-\alpha)]}^{\text {diff }}
\end{array}\right], \\
\Pi_{i} & =\left[\begin{array}{cc}
\Pi_{11_{i[\alpha \times \alpha]}} & 0_{[\alpha \times(n-\alpha)]} \\
\Pi_{21_{i[(n-\alpha) \times \alpha]}} & \Pi_{22_{i}[(n-\alpha) \times(n-\alpha)]}
\end{array}\right],
\end{aligned}
$$

with $i \in \Sigma$ and $\alpha<n$ independent of mode $i$.

The following theorem shows that under particular conditions a partial averaging result can be obtained.

Theorem 32 Consider the regular switched DAE (18) with periodic switching signal $\sigma$ with period $p>0$ given by (5)-(6) and initial condition $x\left(0^{-}\right)=x_{0}$, and consider the partial averaged model (62)-(64). Assume that the following conditions hold.

(i) The matrix pairs $\left(E_{i}, A_{i}\right)$ are regular and (22) holds $\forall i \in \Sigma$.

(ii) The corresponding consistency projectors $\Pi_{i}$, flow matrices $A_{i}^{\text {diff }}$ and $B_{i}^{\text {diff }}$ are in the form of (65).

(iii) $\forall i \in \Sigma$

$$
\begin{aligned}
& \operatorname{im} \Pi_{\cap_{P}} \subseteq \operatorname{im} \Pi_{11_{i}} \\
& \operatorname{ker} \Pi_{\cap_{P}} \supseteq \operatorname{ker} \Pi_{11_{i}} .
\end{aligned}
$$

(iv) The matrix $\prod_{i}^{\mathrm{q}} \Pi_{22_{i}}$ is a projector.

(v) $\Pi_{22_{i}} \Pi_{21_{i-1}}=0, \forall i \in \Sigma$ with $\Pi_{21_{0}}:=\Pi_{21_{\mathrm{q}}}$.

Denote by $x_{\sigma, p}(t)$ the (in general discontinuous) impulsefree part of the (in general distributional) solution of (18) and let $x_{\mathrm{pav}}(t)$ be the solution of the switched partial averaged system (62). Consider an arbitrary constant $\Delta>p$, then

$$
x_{\sigma, p}(t)-x_{\text {pav }}(t)=\mathrm{O}(p),
$$

$\forall t \in[p, \Delta]$

To prove the result we introduce the following Lemma.

Lemma 33 Consider the regular switched DAE (18) with periodic switching signal $\sigma$ with period $p>0$ given by (5)-(6) and initial condition $x\left(0^{-}\right)=x_{0}$, satisfying assumptions (i), (ii) and (iii) of Theorem 32. Let $A_{\text {pav }}$ and $B_{\text {pav }}$ given by (64). Consider the following averaged system

$$
\begin{aligned}
\dot{z}_{\text {pav }}(t) & =A_{\text {pav }} z_{\text {pav }}(t)+B_{\text {pav }} u(t) \\
z_{\text {pav }}(0) & =\Pi_{\cap_{p}}\left[I_{\alpha} 0\right] x_{0} .
\end{aligned}
$$

Denote by $x_{\sigma, p}(t)$ the (in general discontinuous) impulsefree part of the (in general distributional) solution of (18) 
and let $z_{\mathrm{pav}}(t)$ be the solution of the averaged system (68). Consider an arbitrary constant $\Delta>p$, then

$$
\left[I_{\alpha} 0\right] x_{\sigma, p}(t)-z_{\text {pav }}(t)=\mathrm{O}(p)
$$

$\forall t \in[p, \Delta]$.

PROOF. Let $z:=\left[I_{\alpha} 0\right] x$, then assumption (ii) implies that $z$ is governed by the following switched ODE with jumps:

$$
\begin{aligned}
\dot{z}(t) & =A_{11_{i}} z(t)+B_{\alpha_{i}}^{\mathrm{diff}} u(t), \quad t \in\left(s_{k, i}, s_{k, i+1}\right), \\
z\left(s_{k, i}^{+}\right) & =\Pi_{11_{i}} z\left(s_{k, i}^{-}\right) \\
z(0-) & =\left[\begin{array}{ll}
I_{\alpha} & 0
\end{array}\right] x_{0}
\end{aligned}
$$

Invoking the property of $\Pi_{11_{i}}$ the proof follows by extending the Remark 21 to the case of non-homogeneous systems.

Proof of Theorem 32 By decomposing $x_{\text {pav }}(t)=$ $\left[z_{\text {pav }}(t) y_{\text {pav }}(t)\right]^{\top}$ and $x_{\sigma, p}(t)=\left[z_{\alpha}(t) y(t)\right]^{\top}$, we can define the error variables $w_{y}=y-y_{\mathrm{pav}}$ and $w_{z}=z-z_{\mathrm{pav}}$. Then the proof is a straightforward combination of Lemma 33 and Lemma 8.

Example 34 Consider the switched DAE in Example 31, it seems possible to construct a partial averaged model. Indeed, by analyzing the consistency projectors it can be seen that the assumptions on the structure, (iii), (iv) and (v) of Theorem 32 are satisfied, assuming $\alpha=3$. Furthermore analyzing the structures of the following flow matrices

$$
\begin{aligned}
A_{1}^{\text {diff }} & =\left[\begin{array}{cccccc}
-1.24 & 0 & 1.24 & 0 & 0 \\
0 & 6.10 & 0 & 0 & 0 \\
0 & 0 & 0 & 0 & 0 \\
0.12 & 0 & -0.12 & 0 & 0 \\
0 & 0.3 & 0 & 0 & 0
\end{array}\right], & A_{2}^{\text {diff }} & =\left[\begin{array}{cccccc}
-1.53 & -0.15 & 1.12 & 0 & 0 \\
-1.53 & -0.15 & 1.12 & 0 & 0 \\
0 & 0 & 0 & 0 & 0 \\
0.21 & 0.02 & -0.15 & 0 & 0 \\
0.02 & 0 & -0.01 & 0 & 0
\end{array}\right], \\
A_{3}^{\text {diff }} & =\left[\begin{array}{cccccc}
-0.51 & -0.05 & 0 & 0 & 0 \\
-0.51 & -0.05 & 0 & 0 & 0 \\
0 & 0 & 0 & 0 & 0 \\
0.02 & 0 & 0 & 0 & 0 \\
0 & 0 & 0 & 0 & 0
\end{array}\right], & A_{4}^{\text {diff }} & =\left[\begin{array}{ccccc}
0 & 0 & 0 & 0 & -0.0 \\
0 & 6.110 & 0 & 0 & 0 \\
0 & 0 & 0 & 0 \\
0 & 0 & 0 & 0 & 0 \\
0 & 0 & 0 & 0 \\
0 & 0.30 & 0 & 0 & 0
\end{array}\right],
\end{aligned}
$$

we see that (ii) holds. Then it is possible to construct a partial averaged system (62) where the $A_{\mathrm{pav}}$ and $\Pi_{\cap_{p}}$ are given by

$$
A_{\text {pav }}=\left[\begin{array}{ccc}
-0.82 & -0.08 & 0.79 \\
-0.82 & -0.08 & 0.79 \\
0 & 0 & 0
\end{array}\right] \quad \Pi_{\cap_{p}}=\left[\begin{array}{ccc}
\rho_{1} & \rho_{2} & 0 \\
\rho_{1} & \rho_{2} & 0 \\
0 & 0 & 1
\end{array}\right]
$$

for which (67) holds.

To construct the averaged model the following duty cycles $\left(d_{1}, d_{2}, d_{3}, d_{4}\right)=(0.3,0.4,0.2,0.1)$ have been chosen. By comparing the evolution of the state variables obtained in Example 31 to the ones of the partial averaged model we have that the error between the averaging and the switched solutions decrease by decreasing the switching
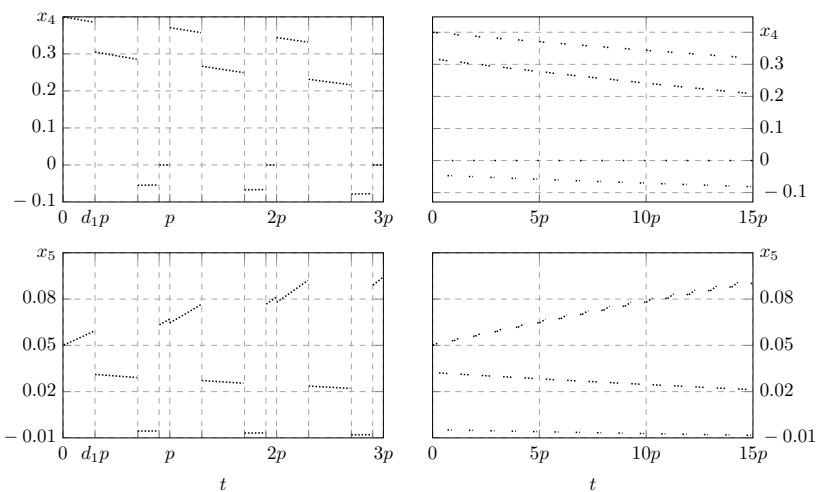

Fig. 6. Averaging dynamics of the state variables (fourth component top, fifth component bottom) for slow switching $(p=0.1 s$, left $)$ and fast switching $(p=0.02 s$, right $)$.

period from $p=0.1 s$ to $p=0.02 s$. The partial averaging dynamics of the fourth and fifth state variables present jumps, as shown in Figure 6. Note that the simulations parameters are the same used in Example 31.

By analyzing the conditions (iii), (iv) of Theorem 32 it can be seen a certain analogy with the case of full averaging in Section 3. The assumption (v) it is not intuitively clearly but is still necessary, as can be shown by the following example of two modes in which it doesn't hold.

Example 35 (Numerical example) Consider the following matrix pairs $\left(E_{i}, A_{i}\right)$ with $i=1,2$

$$
\begin{array}{rlrl}
E_{1} & =\left[\begin{array}{ccc}
1 & 0 & 0 \\
0 & -\frac{3}{4} & 3 \\
3 & -\frac{1}{4} & 1
\end{array}\right], & & A_{1}=\left[\begin{array}{ccc}
0 & 5 & 0 \\
0 & 7 & 0 \\
-3 & 1 & 0
\end{array}\right], \\
E_{2} & =\left[\begin{array}{lll}
1 & 0 & 0 \\
0 & 1 & 1 \\
0 & 0 & 0
\end{array}\right], & A_{2}=\left[\begin{array}{ccc}
5 & -2 & 0 \\
6 & -1 & 0 \\
1 & 3 & 0
\end{array}\right] .
\end{array}
$$

The flow-matrices are the following

$$
A_{1}^{\text {diff }}=\left[\begin{array}{ccc}
-\frac{45}{49} & 0 & 0 \\
\frac{405}{2401} & 0 & 0 \\
-\frac{3711}{9604} & 0 & 0
\end{array}\right], \quad A_{2}^{\text {diff }}=\left[\begin{array}{ccc}
\frac{17}{3} & 0 & 0 \\
-\frac{17}{9} & 0 & 0 \\
\frac{74}{9} & 0 & 0
\end{array}\right],
$$

while the consistency projectors are given by

$$
\Pi_{1}=\left[\begin{array}{ccc}
1 & 0 & 0 \\
-0.18 & 0 & 0 \\
-0.05 & -0.25 & 1
\end{array}\right], \quad \Pi_{2}=\left[\begin{array}{ccc}
1 & 0 & 0 \\
-0.33 & 0 & 0 \\
0.33 & 1 & 1
\end{array}\right]
$$

where one has:

$$
\begin{aligned}
& \Pi_{11_{1}} \Pi_{11_{2}}=\Pi_{11_{2}} \Pi_{11_{1}} \Rightarrow(\text { iii }), \quad \Pi_{11_{1}}, \Pi_{11_{2}} \in \mathbb{R} \\
& \Pi_{22_{1}} \Pi_{21_{2}}=\left[\begin{array}{c}
0 \\
0.42
\end{array}\right] \quad \Pi_{22_{2}} \Pi_{21_{1}}=\left[\begin{array}{c}
0 \\
-0.23
\end{array}\right] .
\end{aligned}
$$

Taking into account the evolution of the first two state variables, it can be seen that the solution of the switched $D A E$ and that of the averaged model become close to each other by decreasing the switching period, as shown in Figure 7. The evolution of the last variable is influenced by (70); it can be seen that the absolute error between the 

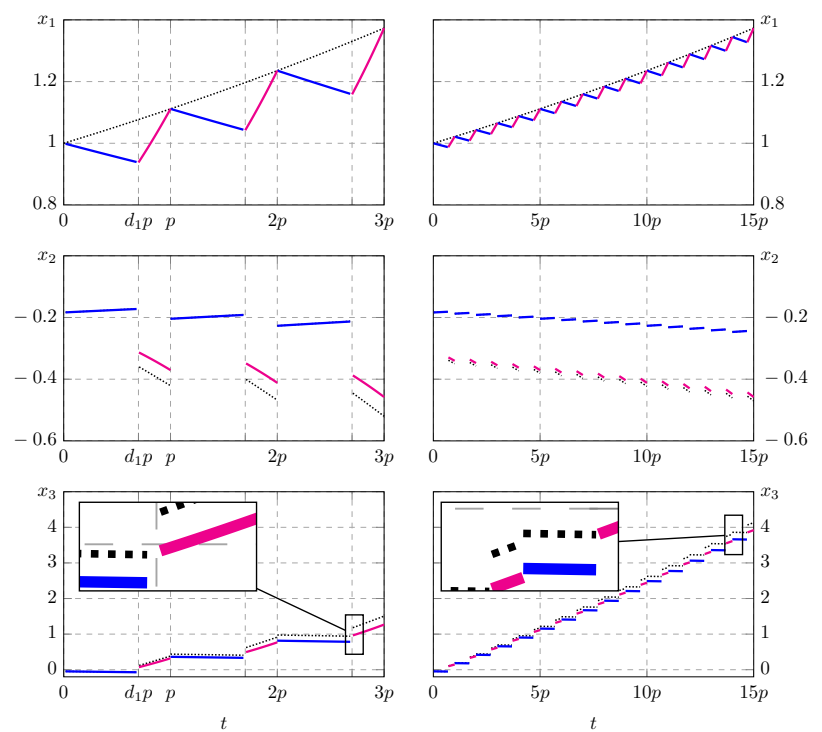

Fig. 7. Evolution of the state variables (first component top, second component middle, third component bottom) of Example 35 for slow switching ( $p=0.1 s$, left) and fast switching $(p=0.02 s$, right). The averaging dynamics are plotted with dotted black lines, while the trajectories of the switched DAE are colored according to the active mode (mode 1 blue, mode 2 magenta).

averaging and the switching dynamics of $x_{3}$ remains the same for both switching periods while the relative error with respect to the value of the state variable decreases by choosing lower values of $p$. Note that the set of consistency projectors is not product bounded as regard the third variable, then the state $x_{3}$ grows unbounded on a fixed time-interval with a switching frequency going to infinity, cf. [20].

\section{Conclusion}

In this paper we have analyzed the averaging technique applied to the switched linear DAEs; an averaged model has been formulated, for which the averaging result holds. In Theorem 18 and Theorem 24 the averaging result is obtained by making assumptions on the image and on the kernel of the consistency projectors. If the averaged model is exponentially stable it exists a switching period $p$ for witch the switched system is also exponentially stable.

We also considered the case in which the state variables present jumps that are independent from the switching period. This state variables cannot be represented in a continuous way but we can still use an averaged model for the remaining state variables. For modeling the whole system jumps are necessary, i.e., we need a partial averaged model; in order to prove the averaging result assumptions on the structures of the consistency projectors and on the flow matrices are also needed. Through the paper different examples are shown to illustrate the applicability of the averaging results for switched DAEs, in particular a switched capacitor circuit has been analyzed.

By analyzing the full and partial averaging results it seems that Theorem 18, Theorem 24 and Theorem 32, can be reformulated for a switching signal $\sigma$ defined in such a way that the duty cycles $d_{i}, i \in \Sigma$ are not fixed in a period, i.e. they can assume different values from one period to another. We can consider a timedependent averaged model, in analogy with the result of the averaging theory for switched ODE, see [13], however the formulation is out of the scope of the paper.

\section{References}

[1] S. Almér and U. Jönsson. Harmonic analysis of pulse-width modulated systems. Automatica, 45(4):851-862, 2009.

[2] T. Berger, A. Ilchmann, and S. Trenn. The quasiWeierstraß form for regular matrix pencils. Lin. Alg. Appl., 436(10):4052-4069, 2012.

[3] A. Van der Schaft and H. Schumacher. An Introduction to Hybrid Dynamical systems. Number 251 in Lecture Notes in Control and Information Sciences. Springer-Verlag, London, 2000.

[4] L. Iannelli, K.H. Johansson, U. Jönsson, and F. Vasca. Subtleties in the averaging of a class of hybrid systems with applications to power converters. Control Engineering Practice, 18(8):961-975, 2008.

[5] L. Iannelli, C. Pedicini, S. Trenn, and F. Vasca. An averaging result for switched DAEs with multiple modes. In Proc. 52nd IEEE Conf. Decis. Control, pages 1378-1383, Florence, Italy, 2013.

[6] L. Iannelli, C. Pedicini, S. Trenn, and F. Vasca. On averaging for switched linear differential algebraic equations. In Proc. 12th European Control Conf., pages 2163-2168, Zürich, Switzerland, 2013.

[7] P. Kunkel and V. Mehrmann. Differential-Algebraic Equations. Analysis and Numerical Solution. EMS Publishing House, Zürich, Switzerland, 2006.

[8] D. Liberzon. Switching in Systems and Control. Systems and Control: Foundations and Applications. Birkhäuser, Boston, 2003.

[9] A. Mironchenko, F. Wirth, and K. Wulff. Stabilization of switched linear differential algebraic equations and periodic switching. IEEE Trans. Autom. Control, 2015. to appear.

[10] E. Mostacciuolo, S. Trenn, and F. Vasca. Partial averaging for switched daes with two modes. In Proc. 14th European Control Conf., Linz, Austria, 2015.

[11] C. Pedicini, L. Iannelli, and F. Vasca. The averaging method for control design and stability analysis of practical switched systems. In IEEE Conf. on Control Appl., part of IEEE Multi-Conference on Systems and Control, pages 1285-1290, Dubrovnik, Croatia, 2012.

[12] C. Pedicini, L. Iannelli, F. Vasca, and U. T. Jönsson. An overview on averaging for pulse-modulated switched systems. In Proc. 50th IEEE Conf. Decis. Control and European Control Conference, pages 1860-1865, Orlando, USA, 2011.

[13] C. Pedicini, L. Iannelli, F. Vasca, and U. T. Jönsson. Averaging for power converters. In Francesco Vasca and Luigi Iannelli, editors, Dynamics and Control of Switched 
Electronic Systems - Advanced Perspectives for Modeling, Simulation and Control of Power Converters, chapter 5, pages 163-188. Springer-Verlag, London, 2012.

[14] M. Porfiri, D. G. Roberson, and D. J. Stilwell. Fast switching analysis of linear switched systems using exponential splitting. SIAM J. Control Optim., 47(5):2582-2597, 2008.

[15] S. Roman. Advanced Linear Algebra. Number 135 in Graduate Texts in Mathematics. Springer-Verlag, New York, USA, third ed edition, 2008.

[16] S. R. Sanders, J. M. Noworolski, X. Z. Liu, and G. C. Verghese. Generalized averaging method for power conversion circuits. IEEE Trans. Power Electron., 6(2):251-259, 1991.

[17] A. Teel, L. Moreau, and D. Nešić. Input-to-state set stability of pulse width modulated systems with disturbances. Syst. Control Lett., 51(1):23-32, 2004.

[18] S. Trenn. Switched differential algebraic equations. In Francesco Vasca and Luigi Iannelli, editors, Dynamics and Control of Switched Electronic Systems - Advanced Perspectives for Modeling, Simulation and Control of Power
Converters, chapter 6, pages 189-216. Springer-Verlag, London, 2012.

[19] S. Trenn. Distributional averaging of switched daes with two modes. submitted for publication, 2015.

[20] S. Trenn and F. Wirth. Linear switched DAEs: Lyapunov exponent, converse Lyapunov theorem, and Barabanov norm. In Proc. 51st IEEE Conf. Decis. Control, pages 2666-2671, Maui, USA, 2012.

[21] W. Wang and D. Nešić. Input-to-state set stability and averaging of linear fast switching systems. IEEE Trans. Autom. Control, 55(5):1274-1279, 2010.

[22] W. Wang, D. Nešić, and A. Teel. Input-to state stability for a class of hybrid dynamical systems via averaging. Math. Control Signals Syst., 23:223-256, 2012. 